\title{
Avionics-Based GNSS Integrity Augmentation for UAS Mission Planning and Real-time Trajectory Optimisation
}

\author{
Roberto Sabatini ${ }^{1}$, Terry Moore ${ }^{2}$ and Chris Hill ${ }^{2}$ \\ ${ }^{1}$ School of Engineering - Aerospace and Aviation Discipline, RMIT University \\ Melbourne, VIC 3000, Australia \\ ${ }^{2}$ Nottingham Geospatial Institute, University of Nottingham \\ Nottingham, NG7 2TU, UK
}

\begin{abstract}
BIOGRAPHIES
Roberto (Rob) Sabatini is a Professor of Aerospace Engineering and Aviation at RMIT University (Australia) with more than 25 years of experience in the aerospace/defence industry and in academia. $\mathrm{He}$ is an expert in Air Traffic Management (ATM), avionics and Unmanned Aircraft Systems (UAS), with specific handson competence in satellite navigation systems, aeronautical Communication, Navigation and Surveillance (CNS), aviation human factors engineering (cognitive HMI, human-machine teaming and trusted autonomy), and multi-sensor data fusion for civil and military applications. Professor Sabatini is a Fellow of the Royal Institute of Navigation.

roberto.sabatini@rmit.edu.au
\end{abstract}

Professor Terry Moore is the Director of the Nottingham Geospatial Institute. He was promoted to the UK's first Chair of Satellite Navigation in 2001 and has extensive research experience in a range of subjects including satellite navigation and positioning, geodesy and orbit determination. He is the founding Director of GRACE, the GNSS Research and Applications Centre of Excellence, which is jointly funded by the University of Nottingham and East Midlands Development Agency. Professor Moore is a Fellow of the Institute of Navigation and of the Royal Institute of Navigation.

terry.moore@nottingham.ac.uk

Doctor Chris Hill is Principal Research Officer in the Nottingham Geospatial Institute. He has extensive R\&D experience in GNSS, Differential GNSS and Integrated Navigation Systems for land, marine and aeronautical applications.

chris.hill@nottingham.ac.uk

\footnotetext{
ABSTRACT

This paper explores the potential of integrating Global Navigation Satellite System (GNSS) Avionics Based Integrity Augmentation (ABIA) functionalities in Unmanned Aerial Systems (UAS) to perform mission planning and real-time trajectory optimisation tasks. In case of mission planning, a pseudo-spectral optimization
}

technique is adopted. For real-time trajectory optimisation a Direct Constrained Optimisation (DCO) method is employed. In this method the aircraft dynamics model is used to generate a number of feasible flight trajectories that also satisfy the GNSS integrity constraints. The feasible trajectories are calculated by initialising the aircraft dynamics model with a manoeuvre identification algorithm. The performance of the proposed GNSS integrity augmentation and trajectory optimisation algorithms was evaluated in representative simulation case studies. Additionally, the ABIA performance was compared with Space-Based and Ground-Based Augmentation Systems (SBAS/GBAS). Simulation results show that the proposed integration scheme is capable of performing safety-critical UAS tasks (CAT III precision approach, UAS Detect-and-Avoid, etc.) when GNSS is used as the primary source of navigation data. There is a synergy with SBAS/GBAS in providing suitable (predictive and reactive) integrity flags in all flight phases. Therefore, the integration of ABIA with SBAS/GBAS is a clear opportunity for future research towards the development of a Space-Ground-Avionics Augmentation Network (SGAAN) for UAS SAA and other safety-critical aviation applications.

\section{Keywords:}

GNSS Integrity, GNSS Augmentation, Avionics Based Integrity Augmentation, Unmanned Aircraft Systems, Trajectory Optimisation, Flight Planning, Caution and Warning.

\section{INTRODUCTION}

Aircraft-Based Augmentation Systems (ABAS), GroundBased Augmentation Systems (GBAS) and Space-Based Augmentation Systems (SBAS) address (using different approaches) all four key aspects of GNSS performance augmentation, namely: accuracy, integrity, availability and continuity [1-3]. The Avionics-Based Integrity Augmentation (ABIA) system was developed to allow real-time avoidance of safety-critical flight conditions and fast recovery of the required navigation performance in case of GNSS data losses. In more detail, the ABIA system addresses all four cornerstones of GNSS integrity augmentation in mission- and safety-critical avionics 
applications: prediction (caution flags), avoidance (optimal flight path guidance), reaction (warning flags) and correction (recovery flight path guidance). Typically, airworthiness requirements impose stringent GNSS data integrity requirements, which cannot be fulfilled by current SBAS and GBAS technologies in some of the most demanding operational tasks (e.g. sense-and-avoid). Therefore, a properly designed Avionics Based Integrity Augmentation (ABIA) system would allow an extended spectrum of autonomous and safety-critical operations [3]. The ABAS approach is particularly well suited to increase the levels of integrity and accuracy (as well as continuity in multi-sensor data fusion architectures) of GNSS in a variety of mission- and safety-critical applications. The ABIA system performs a continuous monitoring of GNSS integrity levels in flight by analysing the relationships between Aircraft $(\mathrm{A} / \mathrm{C})$ manoeuvres and GNSS accuracy degradations or signal losses (Doppler shift, multipath, antenna obscuration, signal-to-noise ratio, jamming, etc.). In case of any detected or predicted integrity threshold violation, the ABIA system provides suitable warning or caution signals to the Automatic Flight Control System (AFCS) and to the ground network, thereby allowing timely correction manoeuvres to be performed.

\section{ABIA SYSTEM}

During flight test activities with GNSS and Differential GNSS (DGNSS) systems [4, 5], it was observed that one or more of the following conditions was prone to cause navigation data outages or severe performance degradations: antenna obscuration, bad satellite geometries and low Carrier-to-Noise ratios $\left(\mathrm{C} / \mathrm{N}_{0}\right)$, Doppler shifts, interference and multipath. The last two problems could be mitigated by existing technology solutions (i.e., choosing a VHF/UHF data link, filtering the radio frequency signals reaching the GNSS antenna, identifying suitable locations for the GNSS antenna, etc.). However, there was little one could do in order to prevent critical events during realistic test/training manoeuvres and particular approach procedures (e.g., curved and segmented approaches) performed with high performance military A/C. The ABIA system is capable of alerting the pilot when the critical conditions for GNSS signal loss are likely to occur (within a specified maximum time-toalert). The $\mathrm{A} / \mathrm{C}$ on-board sensors provide information on the $\mathrm{A} / \mathrm{C}$ relevant flight parameters (navigation data, engine settings, etc.) to an Integrity Flag Generator (IFG), which is also connected to the on-board GNSS receiver. Detailed mathematical algorithms have been developed to cope with the main causes of GNSS signal outages and degradation in flight, namely: obscuration, multipath, interference, fading due to adverse geometry and Doppler shift. Adopting these algorithms, the ABIA system is able to provide steering information to the pilot and electronic commands to the A/C flight control system, allowing realtime avoidance of safety-critical flight conditions and fast recovery of the required navigation performance in case of GNSS data losses. This is achieved by implementing both caution (predictive) and warning (reactive) integrity flags, as well as 4-Dimensional Trajectory (4DT) optimisation models suitable for all phases of flight. Therefore, an advanced ABIA system was developed (Fig. 1). In this system, the on-board sensors provide information on the $\mathrm{A} / \mathrm{C}$ relevant flight parameters (navigation data, engine settings, etc.) to an Integrity Flag Generator (IFG), which is also connected to the GNSS system. Using the available data on GNSS and the relevant flight parameters, integrity signals are generated which can be sent to the Unmanned Aircraft System (UAS) Ground Control Station (GCS) or used by a Flight Path Optimisation (FPO) module. This system addresses both the predictive and reactive nature of GNSS integrity augmentation by producing suitable integrity flags (cautions and warnings) in case of predicted/ascertained GNSS data losses or unacceptable signal degradations exceeding the Required Navigation Performance (RNP) specified for each phase of flight, and providing guidance information to the remote pilot/autopilot to avoid further data losses/degradations. To achieve this, the Integrity Flag Generator (IFG) module produces the following integrity flags $[1,2]$ :

- Caution Integrity Flag (CIF): a predictive annunciation that the GNSS data delivered to the avionics system is going to exceed the RNP thresholds specified for the current and planned flight operational tasks (alert status).

- Warning Integrity Flag (WIF): a reactive annunciation that the GNSS data delivered to the avionics system has exceeded the RNP thresholds specified for the current flight operational task (fault status).

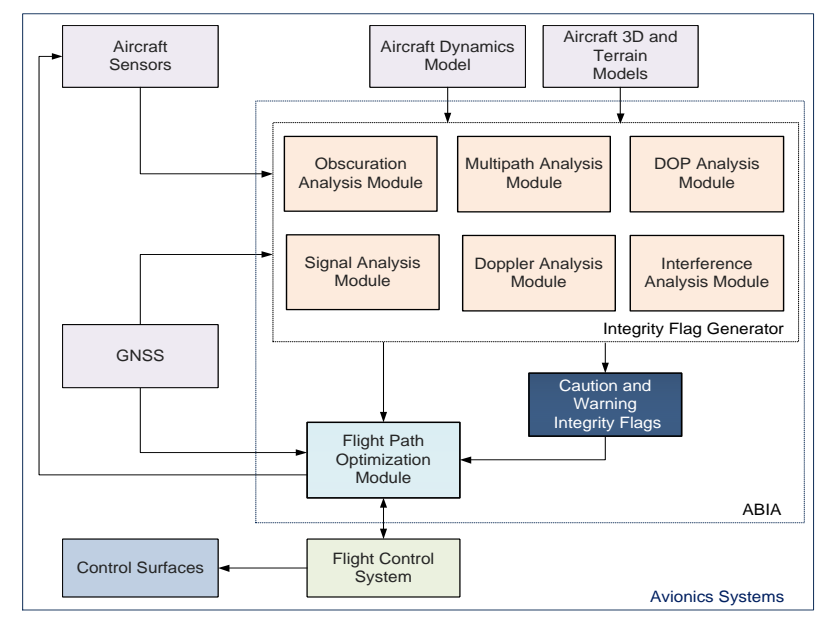

Fig. 1. ABIA system architecture.

The following definitions of Time-to-Alert (TTA) are applicable to the ABIA system [1,2]:

- ABIA Time-to-Caution (TTC): the minimum time allowed for the caution flag to be provided to the user before the onset of a GNSS fault resulting in an unsafe condition.

- ABIA Time-to-Warning (TTW): the maximum time allowed from the moment a GNSS fault resulting in an unsafe condition is detected to the moment that the ABIA system provides a warning flag to the user. 
Based on the above definitions, we can define two separate models for the time responses associated to the Prediction-Avoidance (PA) and Reaction-Correction (RC) functions performed by the ABIA system (Fig. 4-2). The PA time response is given by [1]:

$$
\Delta T_{P A}=\Delta T_{\text {Predict }}+\Delta T_{C-\text { Report }}+\Delta T_{\text {Avoid }}
$$

where:

$$
\begin{aligned}
\Delta T_{\text {Predict }}= & \text { Time required to predict a critical } \\
& \text { condition; } \\
\Delta T_{C-\text { Report }}= & \text { Time required to communicate the } \\
& \text { predicted failure to the FPO module; } \\
\Delta T_{\text {Avoid }}= & \begin{array}{l}
\text { Time required to perform the } \\
\text { avoidance manoeuvre. }
\end{array}
\end{aligned}
$$

In this case, we have $\Delta T_{\text {Avoid }} \leq T T C$. If the available avoidance time $\Delta \mathrm{T}_{\text {Avoid }}$ is not sufficient to perform an adequate avoidance manoeuvre (i.e., $\Delta T_{\text {Avoid }}>T T C$ ), the $\mathrm{A} / \mathrm{C}$ will inevitably encroach on critical conditions causing GNSS data losses or unacceptable degradations. In this case, the RC time response applies:

$$
\Delta T_{R C}=\Delta T_{\text {Detect }}+\Delta T_{W-\text { Report }}+\Delta T_{\text {Correct }}
$$

where:

$$
\begin{aligned}
& \Delta T_{\text {Detect }}= \text { Time required to detect a critical } \\
& \text { condition; } \\
& \Delta T_{W-\text { Report }}= \begin{array}{l}
\text { Time required to communicate the failure } \\
\text { to the FPO module; }
\end{array} \\
& \Delta T_{\text {Correct }}=\begin{array}{l}
\text { Time required to perform the correction } \\
\text { manoeuvre. }
\end{array}
\end{aligned}
$$

In general, we must have $\Delta T_{\text {Detect }}+\Delta T_{W-\text { Report }} \leq T T W$. The RC time response is substantially equivalent to what current GBAS and SBAS systems are capable of achieving. Further progress is possible adopting a suitable algorithm in the IFG module capable of initiating an early correction manoeuvre as soon as the condition $\Delta T_{\text {Avoid }} \leq \mathrm{TTC}$ is violated. In this case, the direct Prediction-Correction (PC) time response would be:

$$
\Delta T_{P C}=\Delta T_{\text {Predict }}+\Delta T_{C-\text { Report }}+\Delta T_{\text {Eraly Correct }}
$$

Therefore, the ABIA system can reduce the time required to recover from critical conditions if the following inequality is verified:

$$
\begin{gathered}
\Delta T_{\text {Eraly Correct }}<T T C+\Delta T_{\text {Detect }}+ \\
\Delta T_{C-\text { Report }}+\Delta T_{\text {Correct }}
\end{gathered}
$$

\section{ABIA INTEGRITY FLAGS}

The ABIA IFG module is designed to provide caution and warning flags (i.e., in accordance with the specified TTC and TTW requirements) in all relevant flight phases. The main causes of GNSS data degradation or signal losses in aviation applications were deeply analysed in [2] and are listed below:

- Antenna obscuration (i.e., obstructions from the wings, fuselage or empennage during maneuvers);
- Adverse satellite geometry, resulting in high Position Dilution of Precision (PDOP);

- Fading, resulting in reduced carrier to noise ratios $\left(\mathrm{C} / \mathrm{N}_{0}\right)$

- Doppler shift, impacting signal tracking and acquisition/reacquisition time;

- Multipath effects, leading to a reduced $\mathrm{C} / \mathrm{N}_{0}$ and to range/phase errors;

- Interference and jamming.

Most GNSS integrity degradations depend on the relative positions of the GNSS receiver antenna and each satellite. The relative motion of the GNSS receiver antenna and the satellites is also crucial. Therefore degradations related to one satellite do not affect the system in the same manner as the others. Specific mathematical models and associated integrity thresholds are introduced for antenna obscuration, Doppler shift, multipath, carrier, interference and satellite geometry degradations. Based on GNSS satellite observations and avionics sensor inputs, the IFG module is capable of detecting adverse conditions leading to unacceptable degradations or losses of satellite signals $[1,2]$. As described in [1], A/C Position, Velocity, Time (PVT) and attitude (Euler angles) data from the on board sensors (i.e., inertial navigation systems, air data computer, etc.), GNSS data (raw measurements and PVT), and Flight Control System (FCS) actuators data are passed to the IFG module. The required navigation, flight dynamics and GNSS constellation data are extracted, together with the relevant information from an $\mathrm{A} / \mathrm{C}$ Three-Dimensional Model (3DM) and from a Terrain and Objects Database (TOD). The philosophy adopted to setup thresholds for the CIF and WIF integrity flags is depicted in Fig. 2.

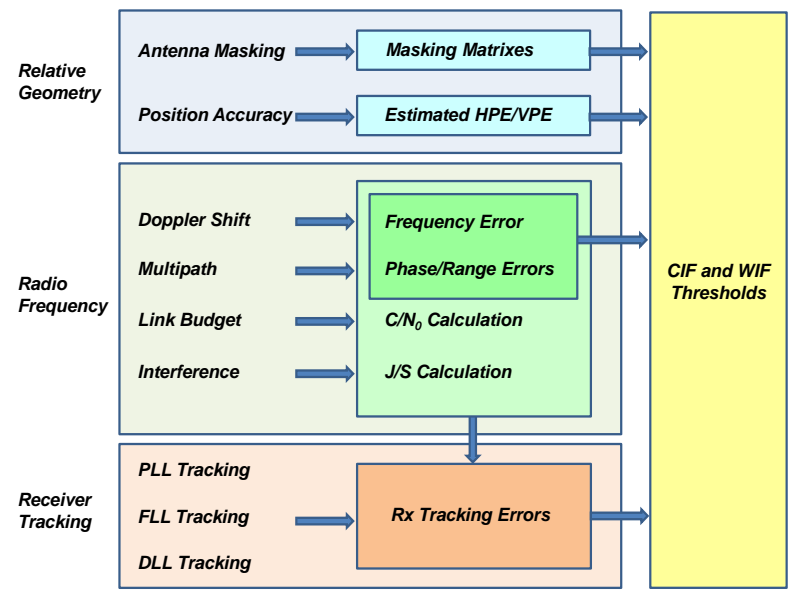

Fig. 2. Integrity flag thresholds criteria.

Integrity flags are generated based on a dedicated error analysis addressing the following aspects of GNSS performance:

- Satellite-A/C (receiver) relative geometry and position errors; 
- Radio frequency (RF) signal errors (i.e., Doppler shift, jamming and multipath);

- Receiver Tracking Errors (RTE).

In particular, the RTE models are used to support the development of robust criteria for the RF signal thresholds, in addition to the criteria based on experimental results (e.g., ground and flight test activities with GNSS). Tables 1 and 2 list the detailed criteria adopted for each CIF and WIF threshold.

Table. 1. CIF criteria [1-3].

\begin{tabular}{|c|c|}
\hline CIF Type & Criteria \\
\hline Masking & $\begin{array}{l}\text { When the current } \mathrm{A} / \mathrm{C} \text { manoeuvre } \\
\text { will lead to less the } 4 \text { satellite in } \\
\text { view, the CIF shall be generated. }\end{array}$ \\
\hline $\begin{array}{l}\text { Satellite } \\
\text { visibility }\end{array}$ & $\begin{array}{l}\text { When one (or more) satellite(s) } \\
\text { elevation angle (antenna frame) is } \\
\text { less than } 10 \text { degrees, the caution } \\
\text { integrity flag shall be generated. }\end{array}$ \\
\hline$D O P$ & $\begin{array}{l}\text { When the } \mathrm{EHE}_{3-\sigma} \text { exceeds the HAL } \\
\text { or the } \mathrm{EVE}_{3-\sigma} \text { exceeds the VAL, the } \\
\text { CIF shall be generated. }\end{array}$ \\
\hline Multipath & $\begin{array}{l}\text { When the ELP exceeds } 0.1 \text { radians, } \\
\text { the caution integrity flag shall be } \\
\text { generated. }\end{array}$ \\
\hline $\begin{array}{l}\text { Tracking } \\
\text { loops }\end{array}$ & $\begin{array}{l}\text { When either } 42.25^{\circ} \leq 3 \sigma_{\mathrm{PLL}} \leq \\
45^{\circ} \text { or } 0.2375 \mathrm{~T} \leq 3 \sigma_{\mathrm{FLL}} \leq \\
0.25 \mathrm{~T} \text { or } 0.05 \mathrm{~d} \leq 3 \sigma_{\mathrm{DLL}} \leq \mathrm{d} \text {, the } \\
\text { CIF shall be generated. }\end{array}$ \\
\hline$C / N_{0}$ & $\begin{array}{l}\text { When the } \mathrm{C} / \mathrm{N}_{0} \text { is less than } 26 \mathrm{~dB}-\mathrm{Hz} \\
\text { the CIF shall be generated. }\end{array}$ \\
\hline Jamming & $\begin{array}{l}\text { When the difference between the } \\
\text { received (incident) jammer power } \\
(\mathrm{dBw}) \text { and the received (incident) } \\
\text { signal power ( } \mathrm{dBw} \text { ) is } 1 \mathrm{~dB} \text { below } \\
\text { the } \mathrm{J} / \mathrm{S} \text { performance of the receiver at } \\
\text { its tracking threshold, the CIF shall } \\
\text { be generated. }\end{array}$ \\
\hline Doppler & $\begin{array}{l}\text { When the } \mathrm{C} / \mathrm{N}_{0} \text { is below } 28 \mathrm{~dB}-\mathrm{Hz} \\
\text { and the signal is lost, the caution } \\
\text { integrity flag shall be generated if the } \\
\text { estimated acquisition time is less } \\
\text { than the application-specific TTA } \\
\text { requirements. }\end{array}$ \\
\hline
\end{tabular}

Table. 2. WIF criteria [1-3].

\begin{tabular}{|c|c|}
\hline WIF Type & Criteria \\
\hline Masking & $\begin{array}{l}\text { When less than } 4 \text { satellites are in } \\
\text { view, the WIF shall be generated. }\end{array}$ \\
\hline $\begin{array}{l}\text { Satellite } \\
\text { visibility }\end{array}$ & $\begin{array}{l}\text { When one (or more) satellite(s) } \\
\text { elevation angle is less than } 5 \text { degrees, } \\
\text { the warning integrity flag shall be } \\
\text { generated. }\end{array}$ \\
\hline$D O P$ & $\begin{array}{l}\text { When the } \mathrm{EHE}_{2-\sigma} \text { exceeds the HAL } \\
\text { or the EVE } E_{2-\sigma} \text { exceeds the VAL, the } \\
\text { CIF shall be generated. }\end{array}$ \\
\hline Multipath & $\begin{array}{l}\text { When the multipath ranging error } \\
\text { shows a sudden increase with the } \\
\text { A/C flying in proximity of the } \\
\text { ground (below } 448.5 \text { metres), the } \\
\text { warning integrity flag shall be } \\
\text { generated. } \\
\text { When the multipath ranging error } \\
\text { exceeds } 2 \text { metres and the A/C flies in } \\
\text { proximity of the ground (below } 500 \\
\text { ft AGL), the warning integrity flag } \\
\text { shall be generated. }\end{array}$ \\
\hline $\begin{array}{l}\text { Tracking } \\
\text { loops }\end{array}$ & $\begin{array}{l}\text { When } 3 \sigma_{\mathrm{PLL}}>45^{\circ} \text { or } 3 \sigma_{\mathrm{FLL}}>1 / \\
4 \mathrm{~T} \text { or } 3 \sigma_{\mathrm{DLL}}>\mathrm{d} \text { the WIF shall be } \\
\text { generated. }\end{array}$ \\
\hline$C / N_{0}$ & $\begin{array}{l}\text { When the } \mathrm{C} / \mathrm{N}_{0} \text { is less than } 25 \mathrm{~dB}-\mathrm{Hz} \\
\text { the CIF shall be generated. }\end{array}$ \\
\hline Jamming & $\begin{array}{l}\text { When the difference between the } \\
\text { received (incident) jammer power } \\
(\mathrm{dBw}) \text { and the received (incident) } \\
\text { signal power ( } \mathrm{dBw} \text { ) is above the } \mathrm{J} / \mathrm{S} \\
\text { performance of the receiver at its } \\
\text { tracking threshold, the WIF shall be } \\
\text { generated. }\end{array}$ \\
\hline Doppler & $\begin{array}{l}\text { When the } \mathrm{C} / \mathrm{N}_{0} \text { is below } 28 \mathrm{~dB}-\mathrm{Hz} \\
\text { and the signal is lost, the warning } \\
\text { integrity flag shall be generated if the } \\
\text { estimated acquisition time exceeds } \\
\text { the application-specific TTA } \\
\text { requirements. }\end{array}$ \\
\hline
\end{tabular}

\section{FLIGHT PATH OPTIMISATION MODULE}

The ABIA FPO module computes the optimal (i.e., GNSS data losses free) flight trajectory. Optimising $\mathrm{A} / \mathrm{C}$ trajectories subject to dynamic, geometric and operational constraints is a well-known optimal control problem that 
can be solved using a variety of direct or indirect methods. All the standard components of an optimization problem are present: the A/C Dynamics Model (ADM) gives the dynamic constraints (allowing the generation of a flyable trajectory); the CIF thresholds and the current GNSS parameters define a certain number of path constraints ensuring that WIF thresholds are not exceeded on the whole trajectory; the boundary conditions include minimum, maximum, initial and final values for the various state and command variables involved in the computation (these are given by the on-board A/C sensors and by the Flight Management System (FMS), which stores the information relative to the initial flight plan). A cost function is implemented to address the minimisation of certain performance criteria. In safety-critical GNSS applications (e.g., curved/segmented precision approaches), all the necessary constraints associated to integrity degradations are included in the path constraints and the trajectory is optimised for minimum time to destination waypoint. However, more complex criteria can be set based on the actual A/C performance parameters (e.g., minimum fuel consumption) or on the characteristics of the mission (i.e., to maximise distance from another $\mathrm{A} / \mathrm{C}$, to minimise the distance from the initial waypoint, etc.).

\section{FLIGHT DYNAMICS MODELS}

As the concept of flight trajectory is deeply related to the dynamics of the body in aerial motion, flight dynamics model implementations are discussed beforehand. The focus of this research is on fixed-wing civil/military $\mathrm{A} / \mathrm{C}$; hence the Six Degree of Freedom (6DOF) and 3 Degree of Freedom (3DOF) models introduced here are specifically tailored to this category of flying platforms. Assuming the $\mathrm{A} / \mathrm{C}$ to be a rigid body with a static mass distribution, a rather accurate model of its flight dynamics can be introduced, which is derived from the equilibrium of forces and momentums along the coordinate axes of a suitable Cartesian reference frame with origin in the centre of mass of the A/C (i.e., body frame). This model involves a high number of parameters to define the properties of inertia, aerodynamic stability and control forces. Adequate experimental and numerical investigations are typically required in order to define the parameters with good precision. For implementation in avionics guidance and control systems (e.g., fly-by-wire flight control systems) and for other applications including flight simulation and trajectory estimation/route planning, A/C flight dynamics are typically described by a set of Differential Algebraic Equations (DAE). The set of DAE and complementary kinematic relations defining the $6 \mathrm{DOF}$ rigid body dynamics of a fixed-wing $\mathrm{A} / \mathrm{C}$ are [6]:

$$
\left[\begin{array}{c}
\dot{u} \\
\dot{v} \\
\dot{w}
\end{array}\right]=\frac{g}{W}\left[\begin{array}{l}
F_{X_{b}} \\
F_{Y_{b}} \\
F_{Z_{b}}
\end{array}\right]+g\left[\begin{array}{c}
-s \theta \\
s \phi c \theta \\
c \phi c \theta
\end{array}\right]+\left[\begin{array}{l}
r v-q w \\
p w-r u \\
q u-p v
\end{array}\right]
$$

$$
\begin{aligned}
& {\left[\begin{array}{c}
\dot{p} \\
\dot{q} \\
\dot{r}
\end{array}\right]=\left[\begin{array}{ccc}
I_{X X_{b}} & 0 & -I_{X Z_{b}} \\
0 & I_{Y Y_{b}} & 0 \\
-I_{Z X_{b}} & 0 & I_{Z Z_{b}}
\end{array}\right]^{-1}} \\
& \cdot\left[\begin{array}{c}
M_{X_{b}}+\left(I_{Y Y_{b}}-I_{Z Z_{b}}\right) q r+I_{X Z_{b}} p q \\
M_{Y_{b}}+\left(I_{Z Z_{b}}-I_{X X_{b}}\right) p r+I_{X Z_{b}}\left(r^{2}-p^{2}\right) \\
M_{Z_{b}}+\left(I_{X X_{b}}-I_{Y Y_{b}}\right) p q-I_{X Z_{b}} q r
\end{array}\right] \\
& {\left[\begin{array}{l}
x_{f} \\
y_{f} \\
z_{f}
\end{array}\right]=\left[\begin{array}{ccc}
c \theta c \psi & s \phi s \theta c \psi-c \phi s \psi & c \phi s \theta c \psi+s \phi s \psi \\
c \theta s \psi & s \phi s \theta s \psi+c \phi c \psi & c \phi s \theta s \psi-s \phi c \psi \\
-s \theta & s \phi c \theta & c \phi c \theta
\end{array}\right] .} \\
& \cdot\left[\begin{array}{c}
u \\
v \\
w
\end{array}\right]+\left[\begin{array}{l}
v_{W X_{f}} \\
v_{W Y_{f}} \\
v_{W Z_{f}}
\end{array}\right] \\
& {\left[\begin{array}{l}
\phi \\
\theta \\
\psi
\end{array}\right]=\left[\begin{array}{ccc}
1 & s \phi s \theta / c \theta & c \phi s \theta / c \theta \\
0 & c \phi & -s \phi \\
0 & s \phi / c \theta & c \phi / c \theta
\end{array}\right] \cdot\left[\begin{array}{l}
p \\
q \\
r
\end{array}\right]}
\end{aligned}
$$

where:

$$
\begin{aligned}
& I_{X X_{b}}, I_{Y Y_{b}}, I_{Z Z_{b}}, I_{X Z_{b}}=\text { non-null components of the } \\
& v_{W X_{f}}, v_{W Y_{f}}, v_{W Z_{f}}=\text { components of the wind vector } \\
& \text { along the three axes of the Earth- } \\
& \text { fixed reference frame }\left[\mathrm{m} \mathrm{s}^{-1}\right] \text {; } \\
& F_{X_{b}}, F_{Y_{b}}, F_{Z_{b}} \quad=\text { components of the aerodynamic } \\
& \text { and propulsive forces acting } \\
& \text { along the three axes of the body } \\
& \text { reference frame }[\mathrm{N}] \text {; } \\
& M_{X_{b}}, M_{Y_{b}}, M_{Z_{b}} \quad=\text { components of the aerodynamic } \\
& \text { and propulsive moments acting } \\
& \text { around the three axes of the body } \\
& \text { reference frame }[\mathrm{N} \mathrm{m}] \text {; } \\
& x_{f}, y_{f}, z_{f}=\text { components of the relative }
\end{aligned}
$$

In particular, Eq. 5 represent the translational dynamics, Eq. 6 the rotational dynamics, Eq. 7 the kinematics and Eq. 8 the Euler rotations of the body frame with respect to the Earth-fixed reference frame. An alternative approach adopted to derive a simplified set of equations for atmospheric flight is based on the approximation of the $\mathrm{A} / \mathrm{C}$ as a point-mass object thereby neglecting the aspects 
associated to its rotational dynamics. The resulting 3DOF models are based on Newton's second law expressed along the coordinate axes of the body frame and on the motion of such frame with respect to an inertial reference frame of convenience. 3DOF models can involve either a constant mass or a variable mass. Models belonging to the first category are adopted when the analysed timeframe is relatively short (so that the fuel consumption may be neglected) or when no fuel is consumed, such as in the case of sailplanes or total engine failures. The 3DOF A/C dynamics model equations can be expressed as follows [7]:

$$
\begin{gathered}
\frac{\mathrm{dV}}{\mathrm{dt}}=\frac{\mathrm{T} \cos \alpha-\mathrm{D}(\mathrm{V}, \mathrm{h}, \mathrm{L})}{\mathrm{m}}-\mathrm{gsin} \gamma \\
\frac{\mathrm{d} \gamma}{\mathrm{dt}}=(\mathrm{T} \sin \alpha+\mathrm{L}) \frac{\cos \phi}{\mathrm{mV}}-\frac{\mathrm{g} \cos \gamma}{\mathrm{V}} \\
\frac{\mathrm{d} \psi}{\mathrm{dt}}=(\mathrm{T} \sin \alpha+\mathrm{L}) \frac{\sin \phi}{\mathrm{mV} \cos \gamma} \\
\frac{\mathrm{d} \Phi}{\mathrm{dt}}=\frac{\mathrm{V} \cos \gamma \cos \psi}{\mathrm{r}_{\mathrm{M}}+\mathrm{h}} \\
\frac{\mathrm{d} \Lambda}{\mathrm{dt}}=\frac{\mathrm{V} \cos \gamma \sin \psi}{\cos \Phi\left(\mathrm{r}_{\mathrm{T}}+\mathrm{h}\right)} \\
\frac{\mathrm{dh}}{\mathrm{dt}}=\mathrm{V} \sin \gamma \\
\frac{d m}{d t}=-F_{F}=-\operatorname{SFC}(V, h) T
\end{gathered}
$$

where:

$\mathrm{m}=\mathrm{A} / \mathrm{C}$ mass $[\mathrm{kg}]$

$\mathrm{V}=$ longitudinal velocity $\left[\mathrm{m} \mathrm{s}^{-1}\right]$;

$\mathrm{T}=$ thrust magnitude $[\mathrm{N}]$;

$\alpha=$ angle of attack [rad];

$\mathrm{h}=$ altitude $[\mathrm{m}]$;

$\mathrm{L} \quad=$ lift magnitude $[\mathrm{N}]$;

$\mathrm{D}=$ drag magnitude $[\mathrm{N}]$;

$\mathrm{g}=$ gravity acceleration $\left[\mathrm{m} \mathrm{s}^{-2}\right]$;

$\gamma=$ flight path angle [rad];

$\phi=$ bank or roll angle [rad];

$\psi=$ heading angle [rad];

$\mathrm{SFC}=$ specific fuel consumption $[\mathrm{kg} / \mathrm{sN}]$;

$\mathrm{FF}=$ fuel flow $\left[1 \mathrm{~s}^{-1}\right]$;

$\Phi=$ geodetic latitude $[\mathrm{rad}]$

$\Lambda=$ geodetic longitude [rad];

$\mathrm{r}_{\mathrm{m}}=$ meridional radius of curvature $[\mathrm{m}]$;

$\mathrm{r}_{\mathrm{t}}=$ transverse radius of curvature $[\mathrm{m}]$.

Key assumptions adopted in the model formulation are:

- Earth's shape is approximated as an ellipsoid using WGS-84 parameters.

- The atmosphere is considered at rest relatively to the earth.

- A standard ISA atmospheric model is adopted to describe temperature, pressure and density variations as a function of altitude.
- The $\mathrm{A} / \mathrm{C}$ is modelled as a rigid body with a vertical plane of symmetry.

- The $\mathrm{A} / \mathrm{C}$ mass reduction in flight is due to fuel consumption only.

- Thrust, aerodynamic forces and weight act on the $\mathrm{A} / \mathrm{C}$ Centre of Gravity (CG).

- All manoeuvres are well coordinated and no sideslip is present.

- The flight is subsonic and the thrust vector is aligned with the longitudinal axis of the A/C (body frame).

The classical formulas for lift and drag are:

$$
\begin{aligned}
& \mathrm{L}=\frac{1}{2} \rho \mathrm{V}^{2} \mathrm{C}_{\mathrm{L}} \mathrm{S} \\
& \mathrm{D}=\frac{1}{2} \rho \mathrm{V}^{2} \mathrm{C}_{\mathrm{D}} \mathrm{S}
\end{aligned}
$$

where $C_{L}$ is the lift coefficient, $C_{D}$ is the drag coefficient, $\mathrm{S}$ is the wing area and $\rho$ is the air density. Both $\mathrm{C}_{\mathrm{L}}$ and $\mathrm{C}_{\mathrm{D}}$ can be obtained from the $\mathrm{A} / \mathrm{C}$ polar curves when available (practical avionics implementations typically adopt lookup tables). Alternatively, the lift and drag coefficients are computed using the available derivatives. For the lift coefficient, the following equation is used:

$$
\mathrm{C}_{\mathrm{L}}=\mathrm{C}_{\mathrm{L} 0}+\mathrm{C}_{\mathrm{L}}^{\alpha} \alpha
$$

where $\mathrm{C}_{\mathrm{L} 0}$ is the zero-alpha lift (i.e., the lift coefficient at zero angle of attack), and $\mathrm{C}_{\mathrm{L}}^{\alpha}$ is the alpha derivative (i.e., the first-order variation of $C_{L}$ with respect to the angle of attack). For the drag coefficient a similar approach can be adopted and the following equation is used:

$$
C_{D}=C_{D 0}+\frac{\left(C_{L}-C_{L m i n}\right)^{2} S}{\pi e_{0} b^{2}}
$$

where $\mathrm{C}_{\mathrm{D} 0}$ is the minimum drag coefficient of the airplane, $C_{\operatorname{Lmin}_{D}}$ is the lift at minimum drag, which is usually but not necessarily equal to $\mathrm{C}_{\mathrm{L} 0}, \mathrm{~b}$ is the wing span and $e_{O}$ is the Oswald efficiency factor (for conventional fixed-wing $\mathrm{A} / \mathrm{C}$ with moderate aspect ratio and sweep, $e_{o}$ is typically between 0.7 and 0.85 ).

$6 \mathrm{DOF}$ and 3DOF rigid-body models are normally unsuitable for the calculation of $\mathrm{A} / \mathrm{C}$ trajectories over extended time periods and this is due to the complexity of the resulting trajectory estimation problem, accumulation of numerical errors, environmental perturbations and the presence of short period modes that are likely to generate instabilities [8]. These models are nevertheless fundamental for the study of $\mathrm{A} / \mathrm{C}$ manoeuvres and more in general for the analysis of $\mathrm{A} / \mathrm{C}$ dynamic stability and control. Therefore, they can be successfully adopted in trajectory prediction/optimisation studies, especially in combination with a careful selection of engine models and path constraints. 


\section{TRAJECTORY OPTIMISATION ALGORITHMS}

As discussed, the IFG outputs (CIFs and WIFs) are used in the FPO module. The key requirement of the FPO module is to generate guidance information that optimize the short-term Autopilot and Flight Director System (A\&FDS) control parameters (e.g., attitude angles and airspeed), as well as the medium/long-term trajectory to be flown. The information update process starts from the current information stored in the Flight Management System (FMS) and produces a new set of geometric/dynamic constraints in all conditions where CIFs are raised (to avoid WIFs). A trajectory optimisation process is then initiated to meet the specified mission objectives and to comply with this new set of constraints. The results obtained from the computation of the optimal trajectory can be utilized as new inputs to the IFG software (both in real-time and mission-planning implementations), so further updates can be performed when needed to prevent the triggering of CIFs and WIFs. From a practical point of view, trajectory optimisation can be defined as the action of finding the inputs to a given system characterised by a set of equations of motion and dynamic constraints, which will maximise or minimise specific parameters (e.g., time, fuel consumption, distance from another $\mathrm{A} / \mathrm{C}$, relative velocities). In most cases, optimisation problems cannot be solved analytically and, therefore, numerical iterative methods need to be used. One of the key challenges of the online trajectory optimisation task is to produce results in real-time (realtime here is intended for the specific application/scenario involved), since the mathematical algorithms and the associated numerical solvers have to be capable of producing accurate and usable outputs in a relatively short time. Offline and online $\mathrm{A} / \mathrm{C}$ trajectory optimisation tasks are typically formulated as Optimal Control Problems (OCP). This is because optimal control theory provides a well-established framework for the determination of dynamic systems optimal trajectories (in a mathematical sense). In the OCP formulation, the trajectory optimisation problem can be analytically stated as follows [9, 10]:

"Determine the states $x(t) \in \mathbb{R}^{n}$, the controls $u(t) \in$ $\mathbb{R}^{m}$, the parameters $p \in \mathbb{R}^{q}$, the initial time $t_{0} \in \mathbb{R}$ and the final time $t_{f} \in \mathbb{R} \mid t_{f}>t_{0}$, that optimise the performance index:

$$
J=\Phi\left[\boldsymbol{x}\left(t_{f}\right), \boldsymbol{u}\left(t_{f}\right), \boldsymbol{p}\right]+\int_{t_{0}}^{t_{f}} \Psi[\boldsymbol{x}(t), \boldsymbol{u}(t), \boldsymbol{p}] d t
$$

subject to the dynamic constraints:

$$
\dot{\boldsymbol{x}}(t)=\boldsymbol{f}[\boldsymbol{x}(t), \boldsymbol{u}(t), t, \boldsymbol{p}]
$$

to the path constraints:

$$
\boldsymbol{C}_{\min } \leq \boldsymbol{C}[\boldsymbol{x}(t), \boldsymbol{u}(t), t ; \boldsymbol{p}] \leq \boldsymbol{C}_{\max }
$$

and to the boundary conditions:

$$
\mathcal{B}_{\min } \leq \mathcal{B}\left[\mathbf{x}\left(\mathrm{t}_{0}\right), \mathbf{x}\left(\mathrm{t}_{f}\right), \mathbf{u}\left(\mathrm{t}_{0}\right), \mathbf{u}\left(\mathrm{t}_{f}\right) ; \mathbf{p}\right] \leq \mathcal{B}_{\max }
$$

where $\Phi$ is the Mayer term and $\Psi$ is the Lagrange term of the cost function expressed in a Bolza form."

OCP can be solved using various methods and the two main categories are: direct methods and indirect methods. The indirect methods are based on calculus of variation. The problem needs to have maximum and minimum boundaries and an initial guess is made between the boundaries, followed by a computation of the different values of the outputs and constraints. The errors between the outputs and the terminal conditions are compared and if the errors are above the specified thresholds, the initial guess is modified accordingly and the process starts again until the errors reach values that meet the threshold conditions. Indirect methods for optimisation present several advantages since the base algorithm can be directly applied to a wide number of optimisation problems with a relatively small number of variables, so that computational requirements are reduced. However, the Hamiltonian systems required by the calculus of variation are often unstable and the error with unknown initial conditions grows rapidly. If the initial conditions estimation differs widely from the actual conditions, the system will converge slowly or even diverge in some cases. Therefore, in the case of complex problems (e.g., high dynamics conditions) a more appropriate method must be employed. In the case of direct methods, the A/C dynamics (continuous) variables are translated into a number of discretised state and command parameters. The problem is then transformed into a finite-dimensional non-linear optimisation problem and the states are implicitly integrated with a quadrature method for a subset of points (nodes) chosen in the main interval defined by the maximum and minimum boundaries. These quadrature methods can be one of the Euler methods, midpoint method, implicit Runge-Kutta method, etc. Direct methods are further divided into direct shooting and collocation methods. Direct shooting methods employ user defined analytical functions to parameterise the states and controls for the entire OCP domain, while collocation methods adopt piecewise polynomial functions to parameterise states and control. The most computationally efficient class of collocation methods adopts linearly independent polynomial functions and is called pseudo-spectral (the spectral decomposition is not performed in the frequency domain but in the time domain, hence the "pseudo" prefix). The main drawbacks of direct methods are a strong accuracy dependency on the selected discretisation functions and the large number of variables introduced by the parameterisation of states and controls, as well as by the implicit integration of subintervals. Therefore, the computational power required for direct methods can be significant. This problem has often being solved considering that the Jacobian and Hessian of the constraints comprise a majority of null derivatives. Compared with indirect methods, in the direct methods there are no means to know how close a given solution is to the optimal solution, which instead is possible in the case of indirect computation. Planning or optimising a new trajectory for an $\mathrm{A} / \mathrm{C}$ is subject to numerous 
objectives and constraints. Those can be derived from Air Traffic Management (ATM) imposed requirements, flight plan/mission objectives, Separation Maintenance and Collision Avoidance (SM/CA), and environmental requirements. Thus, the optimisation process needs to find the best trade-off between all objectives subject to the dynamics/operational constraints associated with the platform, the planned mission and the current flight profile/phase. Clearly, different sets of data (from widely differing sources) and significantly different objectives/constraints can be used at the mission planning stage and in real-time flight trajectory optimisation tasks. ABIA offers the advantage of meeting the requirements of strategic and tactical air operation tasks, with a possibility to also enhance the performance of SM/CA systems that rely on GNSS as the primary source of navigation data. These include modern cooperative SM/CA systems (e.g., ADS-B) or non-cooperative sensors integrated with GNSS-driven Guidance, Navigation and Control (GNC) systems. For a practical implementation, some additional considerations have to be made. The new trajectory determined by the FPO module has to be completely flyable by the A/C and the mission (defined in the FMS flight plan) shall not be compromised by the new trajectory. Additionally, the new trajectory shall not lead to other hazards like terrain, traffic or weather. As a consequence, the FPO module has to be designed to allow the dynamic setting of boundary conditions for the entire set of variables involved. From the discussion above the following key requirements are derived:

The FPO module shall react to any CIF/WIF triggering as follows:

- Initiating an early-correction loop that prevents the occurrence of a WIF, as soon as CIF is generated.

- Initiating an immediate (emergency) correction in the unlikely event of a WIF not preceded by a CIF.

As soon as activated, the FPO module shall set dynamic constraints that allow the computation of an optimal flight trajectory that prevents the triggering of new CIFs/WIFs and that minimises the deviations from the original $\mathrm{A} / \mathrm{C}$ trajectory (e.g., FMS flight plan).

\section{CONSTRAINTS AND BOUNDARY CONDITIONS}

Most GNSS integrity degradations depend on the relative positions of the GNSS receiver antenna and each satellite. The relative motion of the GNSS receiver antenna and the satellite is also crucial. Therefore degradations related to one satellite do not affect the system in the same manner as the others. An analysis of the different type of degradations results in inferring that a common criterion based on satellite elevation variation in the body frame can be adopted as a geometric constrain in the trajectory optimisation process. This applies to all degradations except Doppler shift (so, for this phenomenon a separate analysis is required). The elevation and azimuth angles in body frame (blue) are depicted in Fig. 3 where $P_{S}$ is the satellite position, $\mathrm{P}_{\mathrm{SXY}}$ is the satellite position in $\mathrm{XY}$ frame, $\mathrm{R}$ is the line of sight vector, $\mathrm{E}$ is the elevation angle and $\mathrm{Az}$ is the azimuth angle. The right-hand rule gives the direction of rotation of both elevation and azimuth angles. Considering the top view of the $\mathrm{A} / \mathrm{C}$, when the elevation angle increases in the positive direction (going up), the azimuth is rotating in a clockwise direction. The elevation angle in the body frame is computed using the simple trigonometry relation:

$$
E=\sin ^{-1}\left(\frac{R_{z}}{|R|}\right)
$$

where $R_{Z}$ is the Z-axis component of the line of sight vector. In order to be used as a dynamic constraint for trajectory optimisation, the elevation angle to each satellite is associated to Euler angles by converting the Line-of-Sight (LOS) vector from the East-North-Up (ENU) reference frame to the body frame. The positions of the $\mathrm{A} / \mathrm{C}$ and satellite are given in the ENU frame, so the LOS vector is computed using:

$$
\vec{R}=\overrightarrow{P_{S}}-\overrightarrow{P_{R}}
$$

where $P_{R}$ is the receiver position vector in the ENU frame.

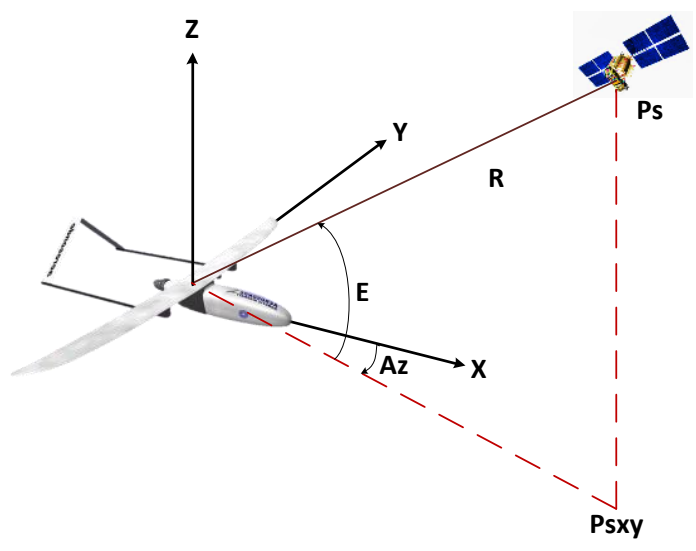

Fig. 3. Elevation and azimuth in body frame

The conversion in body frame is obtained using the matrix:

$C_{B O D Y}^{E N U}=\left[\begin{array}{ccc}C_{\mu} C_{\psi}+S_{\mu} S_{\theta} S_{\psi} & -C_{\mu} S_{\psi}+S_{\mu} S_{\Gamma} C_{\psi} & -S_{\mu} C_{\theta} \\ C_{\theta} S_{\psi} & C_{\theta} C_{\psi} & S_{\theta} \\ S_{\mu} C_{\psi}-C_{\mu} S_{\theta} S_{\psi} & -S_{\mu} S_{\psi}-C_{\mu} S_{\theta} C_{\psi} & C_{\mu} C_{\theta}\end{array}\right]$

where:

$\mathrm{S}_{\theta}=$ sine of the pitch angle;

$\mathrm{C}_{\theta}=$ cosine of the pitch angle;

$\mathrm{S}_{\mu}=$ sine of the bank angle;

$\mathrm{C}_{\mu}=$ cosine of the bank angle;

$\mathrm{S}_{\psi}=$ sine of the yaw angle;

$\mathrm{C}_{\psi}=$ cosine of the yaw angle.

The magnitude of the Doppler shift produce in the signal received from to the $n$th satellite is a function of the relative velocity measured along the satellite-A/C LOS. The magnitude of the Doppler shift can be calculated by:

$$
\Delta f_{n}=f\left(\frac{\left|\overrightarrow{v_{n}}\right| \mp\left|\overrightarrow{v_{a}}\right|}{c}\right) \cos \chi_{n}
$$


where:

$\overrightarrow{v_{n}}=\mathrm{n}^{\text {th }}$ satellite velocity component along the LOS;

$\overrightarrow{v_{a}}=\mathrm{A} / \mathrm{C}$ velocity projection along the LOS;

$c=$ speed of light $\left[\mathrm{m} \mathrm{s}^{-1}\right]$;

$f=$ GNSS signal frequency $[\mathrm{Hz}]$

$\chi_{n}=$ angle between the $\mathrm{A} / \mathrm{C}$ velocity vector and the $\mathrm{n}^{\text {th }}$ satellite LOS.

When the Doppler CIF thresholds are exceeded, the FPO module initiate an optimisation process aiming to avoid any further observed increase in Doppler shift. In particular, the implemented algorithm studies the variations of $\overrightarrow{v_{n}}$ and $\overrightarrow{v_{a}}$ for each tracked satellite and imposes geometric constraints to the trajectory that are accounted for in the trajectory optimisation process. With reference to the geometry illustrated in Fig. 3, the following trigonometric relationship holds true:

$$
\left(\vec{v} \cos \chi_{n}\right) \sin E_{n}=\vec{v} \cos \chi_{n}{ }^{\prime}
$$

where:

$\vec{v} \quad=\mathrm{A} / \mathrm{C}$ velocity vector;

$E_{n}=$ elevation angle of the $\mathrm{n}^{\text {th }}$ satellite;

$\chi_{n}=$ relative bearing of the $\mathrm{A} / \mathrm{C}$ to the satellite;

$\chi_{n}{ }^{\prime}=$ azimuth of the LOS projection in the antenna plane.

Reductions of elevation angles lead to increases in Doppler shift, while $\chi_{\mathrm{n}}{ }^{\prime}$ drives increments or decrements in Doppler shift depending on the size of the angle and the direction of the A/C velocity vector. From Eq. (27) and Eq. (28), we can determine the A/C-satellite relative geometric conditions that maximise or minimise Doppler shift. In particular, combining the two equations we obtain:

$$
\Delta f_{n}=f\left(\frac{\left|\overrightarrow{v_{n}}\right| \mp\left|\overrightarrow{v_{a}}\right|}{c}\right) \frac{\cos \chi_{n^{\prime}}}{\sin E_{n}}
$$

Eq. (29) shows that both the elevation angle of the satellite and the $\mathrm{A} / \mathrm{C}$ relative bearing to the satellite affect the magnitude of the Doppler shift. In particular, reductions of $E_{n}$ lead to increases in Doppler shift, while $\chi_{n}{ }^{\prime}$ drives increments or decrements in Doppler shift depending on the size of the angle and the direction of the $\mathrm{A} / \mathrm{C}$ velocity vector.

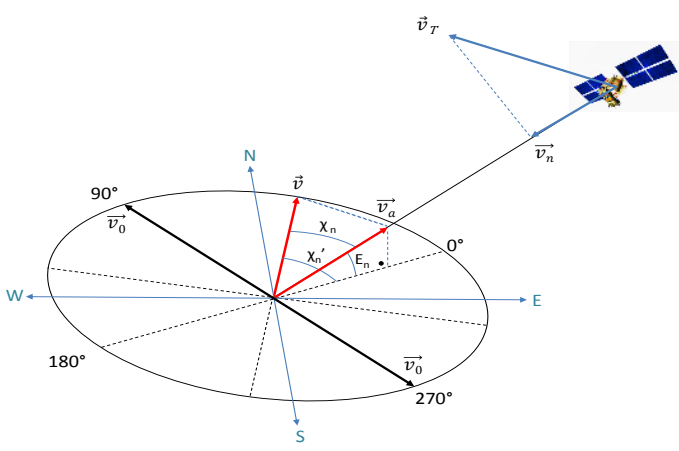

Fig. 4. Reference geometry for Doppler shift analysis.

By inspecting Fig. 4, it is evident that a relative bearing of $90^{\circ}$ and $270^{\circ}$ would lead to a null Doppler shift as in this case there is no component of the $\mathrm{A} / \mathrm{C}$ velocity vector $\left(\overrightarrow{v_{0}}\right.$ in this case) in the LOS to the satellite. However, in all other cases (i.e., $\vec{v} \neq \overrightarrow{v_{0}}$ ), such a component would be present and this would lead to increments or decrements in Doppler shift depending on the relative directions of the vectors $\vec{v}$ and $\overrightarrow{v_{n}}$. This fact is better shown in Fig. 5, where we assume steady flight without loss of generality.

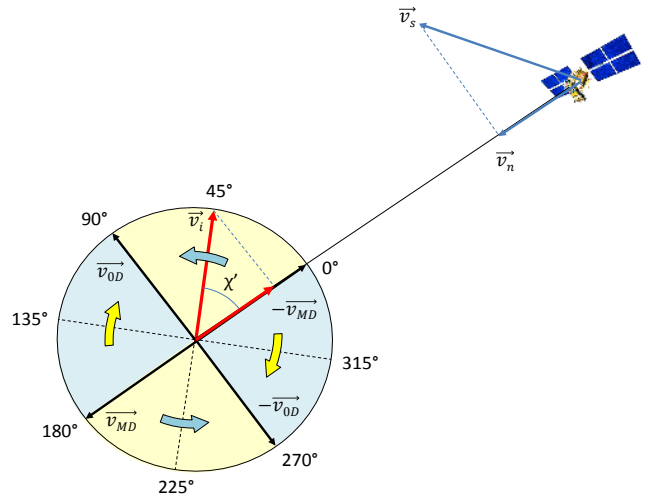

Fig. 5. Reference geometry for Doppler shift manoeuvre corrections

As the time required for typical $\mathrm{A} / \mathrm{C}$ heading change manoeuvres is much shorter than the timeframe associate to significant satellite constellation changes, each satellite can be considered stationary in the body reference frame of the manoeuvring aircraft. Therefore, during manoeuvres leading to Doppler CIFs, we can consider an initial aircraft velocity vector $\overrightarrow{\mathrm{v}_{1}}$ and define path constraints that avoid the Doppler WIF. This can be done by imposing that the aircraft increases the heading rates towards the directions $\overrightarrow{v_{0}}$ or $-\overrightarrow{v_{0}}$ and minimises the heading rates towards the directions $\overrightarrow{\mathrm{V}_{\mathrm{M}}}$ and $-\overrightarrow{\mathrm{V}_{\mathrm{M}}}$. The choice of $\overrightarrow{v_{0}}$ or $-\overrightarrow{v_{0}}$ is based simply on the minimum required heading change (i.e., minimum required time to accomplish the correction). Regarding the elevation angle (E) dependency of Doppler shift, Eq. (29) shows that minimising the elevation angle becomes an objective also in terms of Doppler trajectory optimisation.

\section{COST FUNCTIONS}

The selection of the optimal trajectory is based on minimising a cost function of the form [11]:

$$
J=w_{t} \cdot \mathrm{t}+w_{f} \int[S F C \cdot T(t)] d t
$$

where SFC $\left[\mathrm{kg} \mathrm{N}^{-1} \mathrm{~s}\right]$ is the specific fuel consumption, $\mathrm{T}(\mathrm{t})$ is the thrust profile and $\left\{\mathrm{w}_{\mathrm{t}}, \mathrm{w}_{\mathrm{f}}\right\}$ are the weightings attributed to time and fuel minimisation objectives. In safety-critical UAS applications, this cost function can be expanded to include other parameters such as the distance of the host $\mathrm{A} / \mathrm{C}$ from the avoidance volume associated with a ground obstacle or a conflicting air traffic [12]:

$$
\begin{gathered}
J=w_{t} \cdot \mathrm{t}_{S A F E}+w_{f} \int[S F C \cdot T(t)] d t-\mathrm{w}_{d} \\
\cdot D_{\text {min }}-w_{i d} \cdot \int D(t) d t
\end{gathered}
$$

where $D(t)$ is the estimated distance of the generated avoidance trajectory points from the avoidance volume 
associated with the obstacle, $D_{\min }=\min [\mathrm{D}(\mathrm{t})]$ is the estimated minimum distance of the avoidance trajectory from the avoidance volume, $t_{S A F E}=\left.t\right|_{D_{\min }}$ is the time at which the safe avoidance condition is successfully attained and $\left\{\mathrm{w}_{\mathrm{t}}, \mathrm{w}_{\mathrm{f}}, \mathrm{w}_{\mathrm{d}}, \mathrm{w}_{\mathrm{id}}\right\}$ are the weightings attributed to time, fuel, distance and integral distance respectively. In time-critical avoidance tasks appropriate higher weightings are used for time and distance cost elements and an automated gain control function can be implemented taking into account the host $\mathrm{A} / \mathrm{C}$-obstacle relative dynamics.

\section{MISSION PLANNING OPTIMISATION}

Based on the literature review [9-11], the Radau pseudospectral method was selected for the ABIA mission planning implementation (offline IFG and FPO modules). This widely used methods employ orthogonal collocation Gaussian quadrature implicit integration, where collocation is performed at the Legendre-GaussRadau points. The Generalised Pseudospectral Optimal Control Software, version 2 (GPOPS-II) was chosen for ABIA due to its availability in the public domain and its documented suitability for aerospace applications. GPOPS-II is implemented as a MATLAB toolbox were the user defines the dynamics/path constraints, the boundary conditions and the cost functions that apply to a specific OCP. The user can also define a number of parameters used in the optimisation process, including the quadrature mesh characteristics, the maximum number of iterations and the numerical differentiation method. Further details about GPOPS-II and some examples of aerospace OCPs can be found in (Patterson et al., 2014). The suitability of these techniques for ATM and Air Traffic Flow Management (ATFM) strategic and tactical operations has been demonstrated in recent research [13].

\section{REAL-TIME OPTIMISATION}

For real-time FPO module implementations, a Direct Constrained Optimisation (DCO) method is implemented. In this method the aircraft dynamics model is used to generate a number of feasible flight trajectories that also satisfy the GNSS constraints. The feasible trajectories are calculated by initialising the aircraft dynamics model with a Manoeuvre Identification Algorithm (MIA). The MIA allows identifying a sub-set of ADM equations (and the associated boundary conditions of states and controls) that must be integrated to predict future states and to determine the optimal controls that minimise the cost functions. A schematic representation of the FPG module DCO implementation is shown in Fig. 6. Although this method does not implement an iterative algorithm that converges to the mathematical optimum, it is preferred for real-time safety-critical applications due to its robustness, much reduced complexity and faster convergence rate. Additionally, the DCO prevents problems of nonconvergence or divergence frequently experienced in OCPs for highly non-linear dynamic systems. In the ABIA FPG implementation, the DCO algorithm is designed so that the deviations from the pre-planned flight trajectory (e.g., FMS flight plan) are minimised. This is achieved by introducing additional geometric (path) constraints in the process and implementing a Bézier approximation curve algorithm to guarantee smoothness of the resulting aircraft trajectory [14].

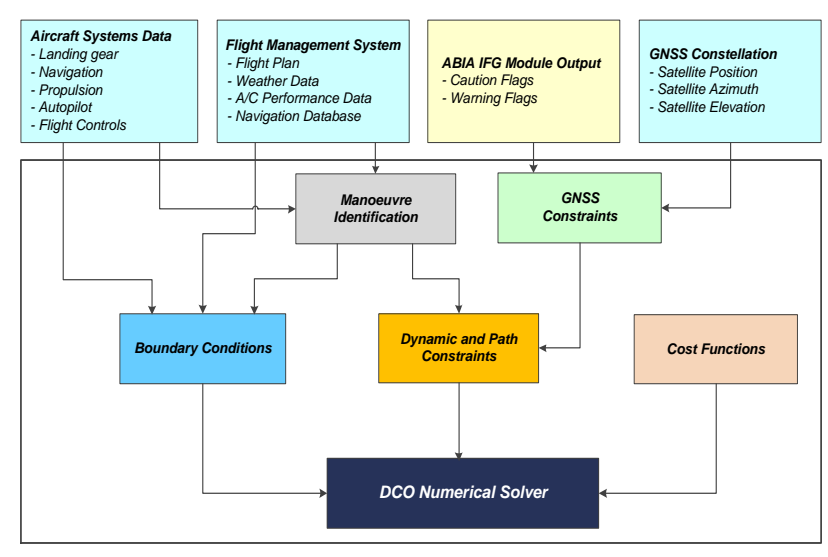

Fig. 6. DCO implementation scheme.

\section{OPTIMISATION CRITERIA}

In both real-time and mission-planning implementations, the flight trajectory optimisation algorithm is initiated when integrity degradations are predicted (CIF generated) by the IFG module. The optimisation criteria adopted in the FPG module are the following:

- With 5 satellites in view, the value of $E_{n}$ for each satellite tracked shall be 5 degrees greater than the threshold value causing the activation of any CIF.

- With 4 satellites in view, the value of $E_{n}$ for each satellite tracked shall be 10 degrees greater than the threshold value causing the activation of any CIF.

- If the CIF is not due to Doppler shift, the minimum elevation limit is set to 5 degrees above the current SV elevation angle.

- If the CIF is due to Doppler shift, the aircraft heading rates are increased towards the directions $\overrightarrow{v_{0}}$ or $-\overrightarrow{v_{0}}$ and reduced in the directions $\overrightarrow{\mathrm{V}_{\mathrm{M}}}$ and $-\overrightarrow{\mathrm{v}_{\mathrm{M}}}$. The choice of $\overrightarrow{\mathrm{v}_{0}}$ or $-\overrightarrow{\mathrm{v}_{0}}$ is based on the minimum required heading change.

- Constrained Geometric Optimisation (CGO) or Pseudospectral Multi-Objective (PMO) Trajectory Optimisation Techniques are used for real-time FPG implementations and for offline mission-planning applications respectively.

The boundary values of each parameter involved in the trajectory optimisation process are obtained from the following sources:

- Navigation Data and Flight Parameters at CIF (NFC), which define the initial conditions when the optimization process in started (i.e., at CIF generation time step). 
- Planned Flight Trajectory (PFT), which define the final condition of the optimization problem. The final condition gives the point when the $\mathrm{A} / \mathrm{C}$ will go back on the initially planned trajectory (e.g., the trajectory stored in the FMS).

- A/C Dynamic Constraints (ADC), which define the minimum and maximum values of the state and control variables.

- Satellite Constellation Data (SCD), which provide the azimuth and elevation boundaries for the path constraints.

\section{SBAS/GBAS INTEGRITY FLAG GENERATION}

The ABIA models can be used to enhance the performance of existing SBAS and GBAS systems. In the proposed implementation, Vertical and Lateral Protection Level (VPL and LPL) for SBAS and GBAS are calculated in line with the performance standards for WAAS and LAAS [1518]. These are compared to the Vertical and Lateral Alert Limits (VAL and LAL) specified for each flight phase (for SBAS) and for each GNSS Landing System (GLS) class (for GBAS). The criteria for producing SBAS/GBAS CIFs and WIFs are listed below:

- When VPL $\mathrm{V}_{\mathrm{SBAS}}$ exceeds VAL or HPL $\mathrm{L}_{\mathrm{SBAS}}$ exceeds HAL, the WIF is be generated.

- When PVPL $\mathrm{GBAS}_{\mathrm{GB}}$ exceeds VAL or PLPL $\mathrm{GBAS}_{\mathrm{GB}}$ exceeds LAL, the CIF is generated.

- When VPL $\mathrm{GBAS}_{\mathrm{GB}}$ exceeds VAL or HPL $\mathrm{HBAS}_{\mathrm{GB}}$ exceeds HAL, the WIF is generated.

As both SBAS and GBAS use redundant GNSS satellite observations to support Fault Detection and Exclusion (FDE) within the GNSS receiver, some additional integrity flag criteria can be introduced. Based on FAA technical standard orders TSO-C145C and TSO-C146C [19], a current generation WAAS-enabled GNSS receiver uses RAIM for instances when the augmentation signal becomes unavailable. In this WAAS/RAIM integration scheme, the minimum number of satellites required for FDE is 6. At present, no information is available regarding the provision of RAIM features within LAASenabled GNSS receivers. Therefore, we can assume the inclusion of a basic form of RAIM within such receiver (i.e., at least 5 satellites are required for FDE). Based on these assumptions, the number of satellites in view can be used to set additional integrity thresholds for SBAS and GBAS:

- When the number of satellites in view is less than 6, the GBAS CIF is generated.

- When the number of satellites in view is less than 5 , the GBAS WIF is generated.

- When the number of satellites in view is less than 7, the SBAS CIF is generated.
- When the number of satellites in view is less than 6, the SBAS WIF is generated.

These new thresholds are well suited for implementation into the ABIA IFG.

\section{SIMULATION CASE STUDIES}

In order to validate the design of the ABIA IFG module and the synergies with GBAS and SBAS, some detailed simulation case-studies were performed on AEROSONDE "Laima" UAS platform. All simulated A/C trajectories included the following flight phases and flight legs:

- Takeoff: Straight Climb (SC) leg;

- Route Capture: Turning Climb (TC) leg;

- Cruise Phase: Straigh and Level (SL) and/or Level Turn (LT) legs;

- Initial Descent: Turning Descent (TD) and/or Straight Descent (SD) leg;

- $\quad$ Final Descent: Straight descent leg to Approach (AP).

The duration of each flight leg was defined in accordance with the typical mission profiles of the designated aircraft types. The terrain profile was assumed to be flat and free from man-made features. No jamming sources were considered in the simulation case studies. For the avionics GPS receiver characteristics, we used a C/A code receiver with a flat random vibration power curve from $20 \mathrm{~Hz}$ to $2000 \mathrm{~Hz}$ with amplitude of $0.005 \mathrm{~g}^{2} / \mathrm{Hz}$ and the oscillator vibration sensitivity $S_{v}\left(f_{m}\right)=1 \times 10^{-9}$ parts/g. Additionally, a third-order loop noise bandwidth of $18 \mathrm{~Hz}$ was considered and a maximum LOS jerk dynamic stress of $10 \mathrm{~g} / \mathrm{s}=98 \mathrm{~m} / \mathrm{s}^{3}$ was assumed. Finally, the following simplified antenna gain pattern was adopted:

$$
G_{R}(d B)=7.8659 * \sin E-4.3659
$$

This resulting antenna gain pattern is shown in Fig. 7.

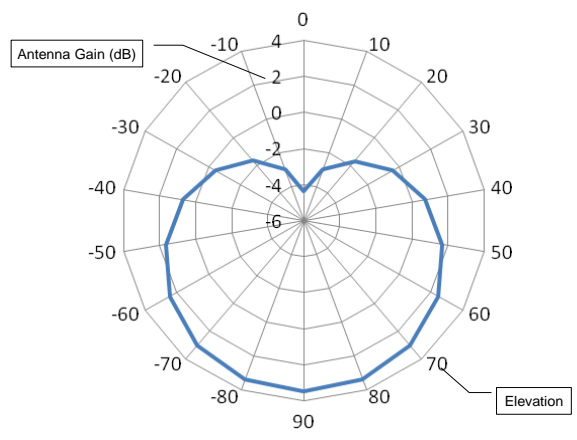

Fig. 7. Simplified GPS antenna gain pattern.

The initial point of the $\mathrm{A} / \mathrm{C}$ trajectory was located at London Heathrow international airport (WGS coordinates: $51^{\circ} 28^{\prime} 39^{\prime \prime} \mathrm{N}, 0^{\circ} 27^{\prime} 41^{\prime \prime} \mathrm{W}$ ) and the GPS constellation available on the 30th of July 2012 (starting at 10:00 a.m.) was simulated using the YUMA almanac data. The CIFs and WIFs relative to antenna masking, 
geometric accuracy degradations, S/N, multipath and Doppler shift were generated. The relevant AEROSONDE geometric parameters were extracted from the literature to draw a detailed 3-D model of the aircraft (Holland et al., 2001), (Burston et al., 2014), (Aircraft Drawings, 2012). The AEROSONDE 3-D CATIA model obtained is shown in Fig. 8.

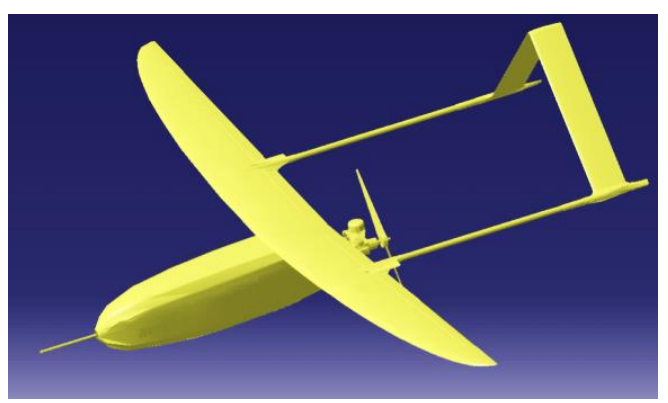

Fig. 8. AEROSONDE 3-D CATIA model.

The aircraft has a wing span of $2.9 \mathrm{~m}$, a length of $2.2 \mathrm{~m}$, a wing area of $0.55 \mathrm{~m}^{2}$ and a Propeller Radius of $0.25 \mathrm{~m}$. The AEROSONDE version considered is equipped with a $24 \mathrm{cc}$ fuel injected premium unleaded gasoline engine and its overall weight is $13-15 \mathrm{~kg}$ (29-33 lbs.) depending on payload, fuel tank and battery configurations. The payload is up to $2 \mathrm{~kg}$ (4.4 lbs.) with full fuel load $(5 \mathrm{~kg})$. The UAV can reach a speed of $80-150 \mathrm{~km} / \mathrm{hr}(50-93$ miles/hour) in cruise and $9 \mathrm{~km} / \mathrm{hour}(6 \mathrm{miles} / \mathrm{hr})$ in climb. The operational range is greater than $3,000 \mathrm{~km}$ with an endurance of 30 hours at $0.1-6 \mathrm{~km}$ altitude (depending on payload). Communication tasks are accomplished by $\mathrm{V} / \mathrm{UHF}$ radio and/or LEO satellites. The location of the AEROSONDE GPS antenna is shown in Fig. 9.

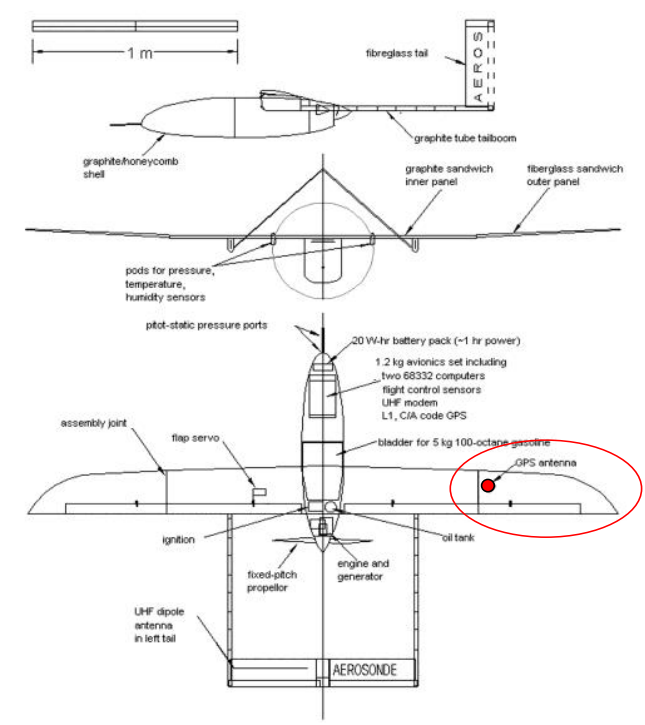

Fig. 9. AEROSONDE UAV antenna location. Adapted from [20].

To speed-up and automate the process of Antenna Masking Matrix (AMM) generation, an Automatic Masking Profile Computation (AMPC) software was developed (Fig. 10).

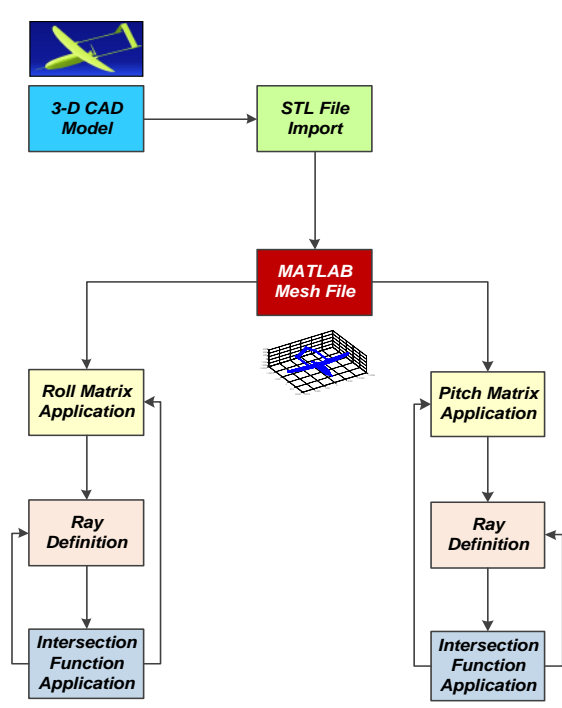

Fig. 10. AMPC logic diagram.

The AMPC software populates a database (look up tables) containing the obscuration information of GNSS satellite signals for different aircraft roll and pitch angles. This is accomplished by implementing two different modules in the AMPC: the first is used to transform the aircrafts CAD model in a mesh of small triangular surfaces that allows straightforward computations of line/surface intersections in a MATLAB ${ }^{\mathrm{TM}}$ environment; the second is used to rotate the aircraft in pitch and roll (bank), and to calculate the intersections between the aircraft structure (i.e., fuselage, wings and tail) and the line-of-sight (LOS) to all satellites in view as illustrated in Fig. 8. After creating the 3-D aircraft surface model, the corresponding CAD file was transformed in a Stereolithography (STL) file format. An STL file is a convenient representation of a complex 3D surface geometry, made by a number of oriented triangles (mesh). Each of these triangles is described by two elements: the first is a unit normal vector to the facet; the second element is a set of three points (listed in counter clockwise order) representing the vertices of the triangle. This representation is ideally suited for the ABIA simulation environment. As an example, the AEROSONDE mesh imported and plotted in MATLAB $^{\mathrm{TM}}$ is illustrated in Fig. 11.

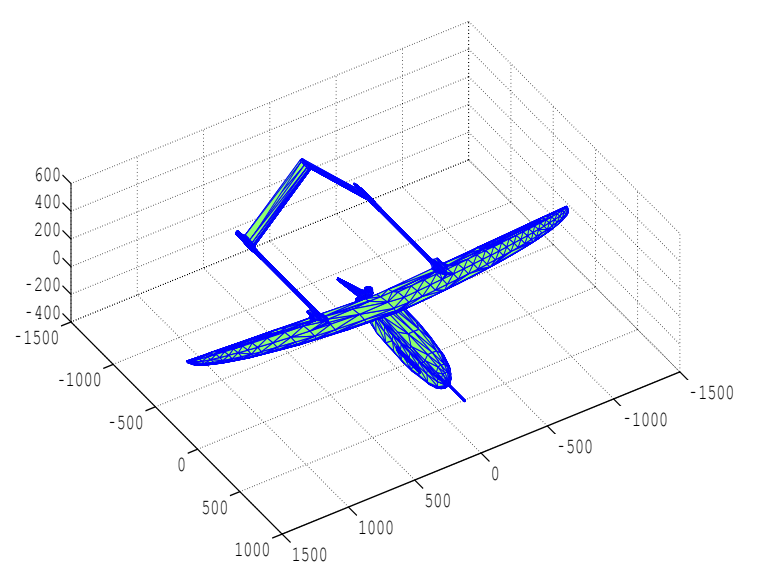

Fig. 11. AEROSONDE mesh in MATLAB ${ }^{\mathrm{TM}}$. 
Using this representation, the AMM is generated calculating all possible intersections of the aircraft body (all triangular surfaces) with the LOS antenna-satellites during pitch and roll motion (Fig. 12).

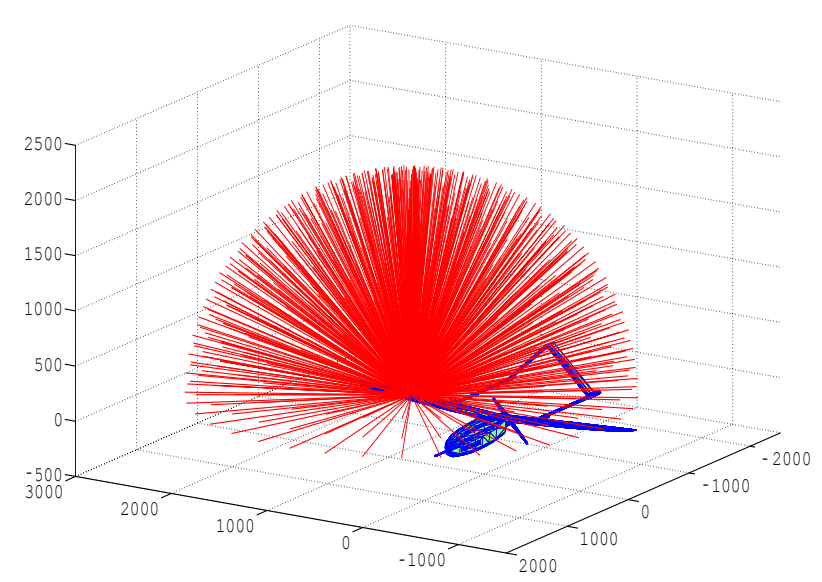

Fig. 12. AEROSONDE masking profile simulation.

The simulated AEROSONDE UAV trajectory, generated using the aircraft 6DOF model [21, 22], is shown in Fig. 13. It includes the seven flight legs described above ( $\mathrm{SC}=$ $450 \mathrm{~s}, \mathrm{TC}=450 \mathrm{~s}, \mathrm{SL}=1300 \mathrm{~s}, \mathrm{LT}=450 \mathrm{~s}, \mathrm{TD}=450 \mathrm{~s}$, $\mathrm{SD}=200 \mathrm{~s}$ and $\mathrm{AP}=100 \mathrm{~s}$ ), for a total of $3400 \mathrm{~s}$.

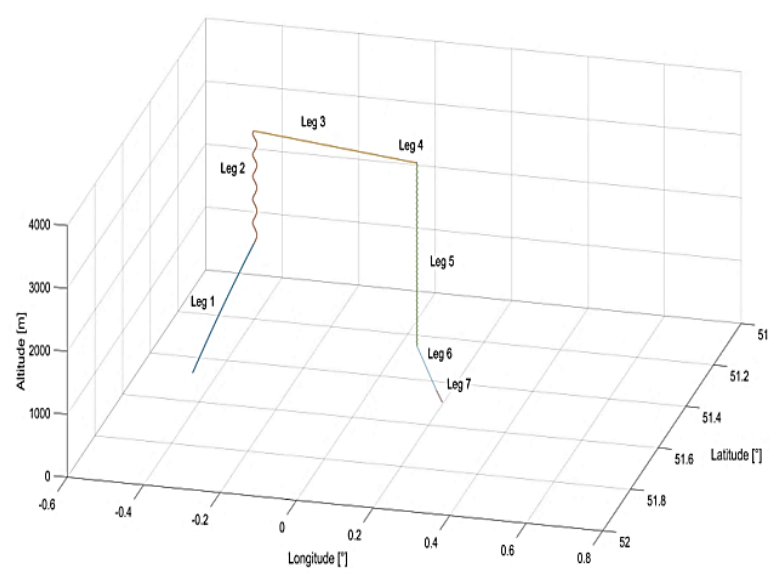

Fig. 13. AEROSONDE UAV simulated trajectory.

The results of the AEROSONDE IFG simulation are listed in Table 3. The CIFs were always triggered at least 2 seconds before the successive WIFs. All CIFs were followed by WIFs leading to $D R_{C I F}$ of $100 \%$. Additionally, all CIFs were followed by a WIF, A total of 13 CIFs were generated and 3 were not associated to WIFs. Therefore, the False Alarm Rate (FAR) was $F A R_{C I F}=23 \%$. These results corroborate the general validity of the models developed for the CIF/WIF thresholds.
Table 3. Integrity Flags for AEROSONDE.

\begin{tabular}{|c|c|c|}
\hline LEG & CIF Time & WIF Time \\
\hline & $2241 \sim 2311 \mathrm{~s}$, & $2259 \sim 2263 \mathrm{~s}, 2273 \sim 2283 \mathrm{~s}$, \\
LT & $2413 \sim 2485 \mathrm{~s}$, & $2432 \sim 2436 \mathrm{~s}, 2446 \sim 2485 \mathrm{~s}$, \\
& $2491 \sim 2650 \mathrm{~s}$ & $2609 \sim 2612 \mathrm{~s}, 2621 \sim 2630 \mathrm{~s}$ \\
\hline & $2688 \sim 2752 \mathrm{~s}$, & \\
TD & $2811 \sim 2881 \mathrm{~s}$, & $2690 \sim 2739 \mathrm{~s}, 2814 \sim 2869 \mathrm{~s}$, \\
& $2944 \sim 3012 \mathrm{~s}$, & $2946 \sim 3003 \mathrm{~s}, 3081 \sim 3100 \mathrm{~s}$ \\
& $3079 \sim 3100 \mathrm{~s}$ & -- \\
\hline SD & --- & $3303 \sim 3400 \mathrm{~s}$ \\
\hline AP & $3301 \sim 3400 \mathrm{~s}$ & \\
\hline
\end{tabular}

Fig. 14 shows the AEROSONDE UAV flight trajectory and illustrates the portion of the TMA where GBAS is available. In this case, GBAS provides information when the AEROSONDE is flying the legs number 5 (TD), 6 (SD) and 7 (AP). The aircraft enters the GLS Coverage Area (GCA) during the TD leg after 2745 seconds from take-off and 205 seconds before landing (end of the simulation). Use of SBAS/GBAS approach modes is assumed in legs 6 (SD) and 7 (FA).

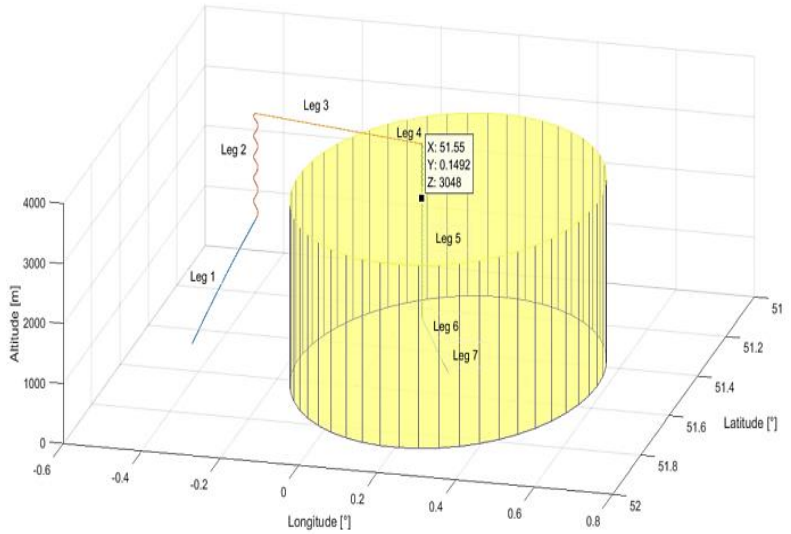

Fig. 14. AEROSONDE UAV simulated trajectory.

The following assumptions are adopted for the SBAS/GBAS simulation and the associated parameters are set in line with the applicable standards [15-18]:

- The SBAS equipment is class 3 and is used for Lateral/Vertical Navigation (LNAV/VNAV) and Localizer Performance with Vertical Guidance (LPV 1/LPV2) operations.

- The Airframe Multipath Designator (AMD) is type A.

- The number of GBAS reference receivers is 4 .

- The GBAS service coverage is $20 \mathrm{NM}$ from the Landing Threshold Point (LTP). 
- GSL class is F, the Airborne Accuracy Designator (AAD) is B, and the Ground Accuracy Designator (GAD) is $B$.

The SBAS WIFs and CIFs recorded during the flight are listed in Table 4.

Table 4. CIF and WIF for SBAS (AEROSONDE).

\begin{tabular}{|c|c|c|c|}
\hline \multicolumn{2}{|c|}{ LEG } & CIF & WIF \\
\hline \multicolumn{6}{|c|}{ LT } & $2201 \sim 2650 \mathrm{~s}$ & $\begin{array}{l}2296 \sim 2358 \mathrm{~s}, \\
2445 \sim 2448 \mathrm{~s}, \\
2453 \sim 2650 \mathrm{~s}\end{array}$ \\
\hline \multirow{2}{*}{ TD } & $2651 \sim 3100 \mathrm{~s}$ & $\begin{array}{c}2701 \sim 2739 \mathrm{~s}, \\
2832 \sim 2869 \mathrm{~s}, \\
3081 \sim 3093 \mathrm{~s}\end{array}$ \\
\hline \multirow{4}{*}{ AP } & L/VNAV & --- & -- \\
\cline { 2 - 4 } & LPV 1 & --- & $3161 \sim 3400 \mathrm{~s}$ \\
\cline { 2 - 4 } & LPV 2 & --- & $3161 \sim 3400 \mathrm{~s}$ \\
\hline
\end{tabular}

The GBAS integrity flags generated during the final portions of the flight are listed in Table 5.

Table 5. CIF and WIF for GBAS (AEROSONDE).

\begin{tabular}{|c|c|c|c|}
\hline \multicolumn{2}{|c|}{ LEG } & CIF & WIF \\
\hline \multicolumn{1}{|c|}{ TD } & $2745 \sim 3100 \mathrm{~s}$ & $3051 \sim 3100 \mathrm{~s}$ \\
\hline \multirow{4}{*}{ AP } & LPV 1 & -- & -- \\
\cline { 2 - 4 } & LPV 2 & --- & -- \\
\cline { 2 - 2 } & CAT I & & \\
\cline { 2 - 2 } & CAT II & $3301 \sim 3400 \mathrm{~s}$ & $3301 \sim 3400 \mathrm{~s}$ \\
\cline { 2 - 2 } & CAT III & & \\
\cline { 2 - 2 } & & & \\
\hline
\end{tabular}

Table 6 lists all CIFs and WIFs generated by SBAS, GBAS and ABIA in the various flight phases.

Based on these simulation case studies, it is evident that the ABIA system works synergically with SBAS and GBAS, enhancing integrity levels in the various flight phases. The ABIA algorithms are capable of generating suitable predictive and reactive flags (CIFs and WIFs) in the same flight phases where SBAS and GBAS are designed to operate but using different principles (i.e., differing input data and integrity models/thresholds). Therefore, the integration of ABIA with SBAS and GBAS is an opportunity for future research towards the development of a Space-Ground-Avionics Augmentation Network (SGAAN) suitable for manned and unmanned aircraft applications and for a variety of mission-critical and safety-critical aviation applications, including flight test, precision approach and automatic landing.
Table.6. CIF and WIF for ABIA, SBAS and GBAS.

\begin{tabular}{|c|c|c|c|}
\hline \multicolumn{2}{|c|}{ LEG } & CIF & WIF \\
\hline \multirow{2}{*}{\multicolumn{2}{|c|}{ LT }} & $\begin{array}{c}\text { ABIA } \\
2241 \sim 2311 \mathrm{~s}, \\
2413 \sim 2485 \mathrm{~s}, \\
3491 \sim 2650 \mathrm{~s}\end{array}$ & $\begin{array}{c}\text { ABIA } \\
2259 \sim 2263 \mathrm{~s}, \\
2273 \sim 2283 \mathrm{~s}, \\
2432 \sim 2436 \mathrm{~s}, \\
2446 \sim 2485 \mathrm{~s}, \\
2609 \sim 2612 \mathrm{~s}, \\
2621 \sim 2630 \mathrm{~s}\end{array}$ \\
\hline & & $\begin{array}{c}\text { SBAS } \\
2201 \sim 2650 \mathrm{~s}\end{array}$ & $\begin{array}{c}\text { SBAS } \\
2201 \sim 2650 \mathrm{~s}\end{array}$ \\
\hline \multirow{3}{*}{\multicolumn{2}{|c|}{ TD }} & $\begin{array}{c}\text { ABIA } \\
2688 \sim 2752 \mathrm{~s}, \\
2811 \sim 2881 \mathrm{~s}, \\
2944 \sim 3012 \mathrm{~s}, \\
3079 \sim 3100 \mathrm{~s}\end{array}$ & $\begin{array}{c}\text { ABIA } \\
2690 \sim 2739 \mathrm{~s}, \\
2814 \sim 2869 \mathrm{~s}, \\
2946 \sim 3003 \mathrm{~s}, \\
3081 \sim 3100 \mathrm{~s}\end{array}$ \\
\hline & & $\begin{array}{c}\text { SBAS } \\
2651 \sim 3100 \mathrm{~s}\end{array}$ & $\begin{array}{c}\text { SBAS } \\
2651 \sim 3100 \mathrm{~s}\end{array}$ \\
\hline & & $\begin{array}{c}\text { GBAS } \\
2745 \sim 3100 \mathrm{~s}\end{array}$ & $\begin{array}{c}\text { GBAS } \\
3051 \sim 3100 \mathrm{~s}\end{array}$ \\
\hline \multirow{6}{*}{$\mathrm{AP}$} & L/VNAV & \multirow{3}{*}{$\begin{array}{c}\text { ABIA } \\
3301 \sim 3400 \mathrm{~s}\end{array}$} & $\begin{array}{c}\text { ABIA } \\
3303 \sim 3400 \mathrm{~s}\end{array}$ \\
\hline & LPV 1 & & \multirow{2}{*}{$\begin{array}{c}\text { SBAS } \\
3161 \sim 3400 \mathrm{~s}\end{array}$} \\
\hline & LPV 2 & & \\
\hline & CAT I & \multirow{3}{*}{$\begin{array}{c}\text { ABIA } \\
3301 \sim 3400 \mathrm{~s} \\
\text { GBAS } \\
3301 \sim 3400 \mathrm{~s}\end{array}$} & \multirow{3}{*}{$\begin{array}{c}\text { ABIA } \\
3303 \sim 3400 \mathrm{~s} \\
\text { GBAS } \\
3301 \sim 3400 \mathrm{~s}\end{array}$} \\
\hline & CAT II & & \\
\hline & CAT III & & \\
\hline
\end{tabular}

To test the FPG module ability to optimise the aircraft flight trajectories in mission-planning and real-time ABIA implementations, a flight segment was extracted from leg 5 (CIFs/WIFs are illustrated in Fig. 15) and used to test the PMO and CGO flight path optimisation techniques. The selected flight segment is shown in Fig. 16.

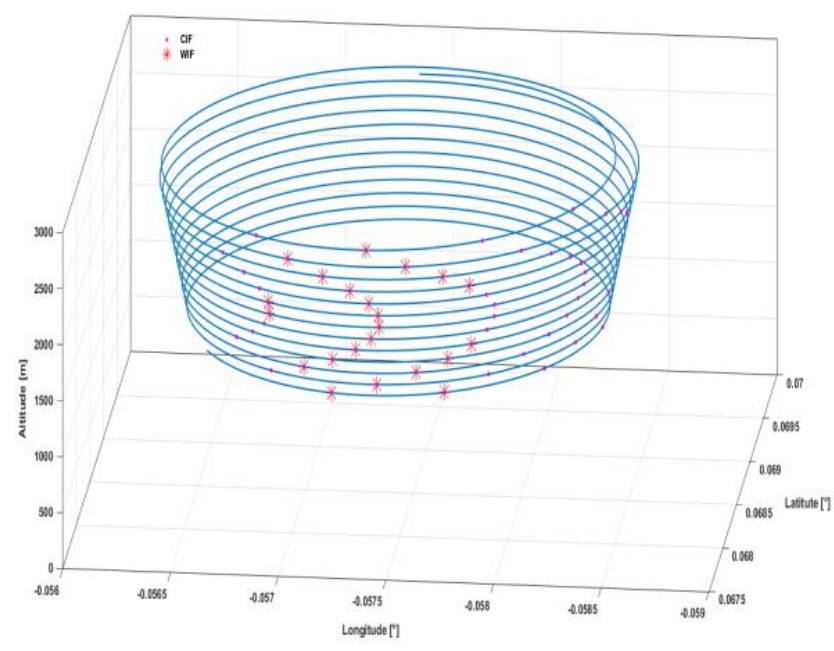

Fig. 15. CIFs and WIFs generated during leg 5 (TD) 


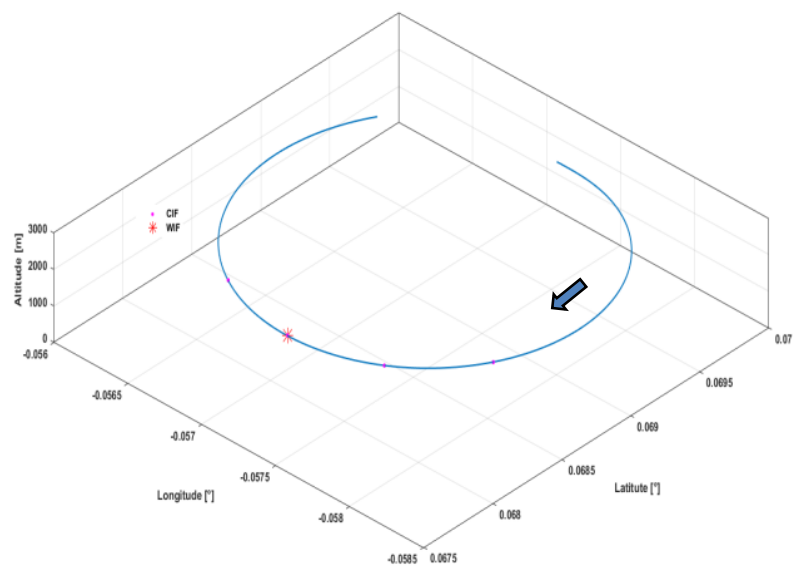

Fig. 16. TD leg segment with CIF and WIF.

Fig. 17 shows the results obtained implementing the PMO technique with a 3DOF dynamic model. In this case, the trajectory optimisation loop took 8.12 seconds to complete in a standard PC equipped with an Intel i7 quadcore processor and 8 GB RAM.

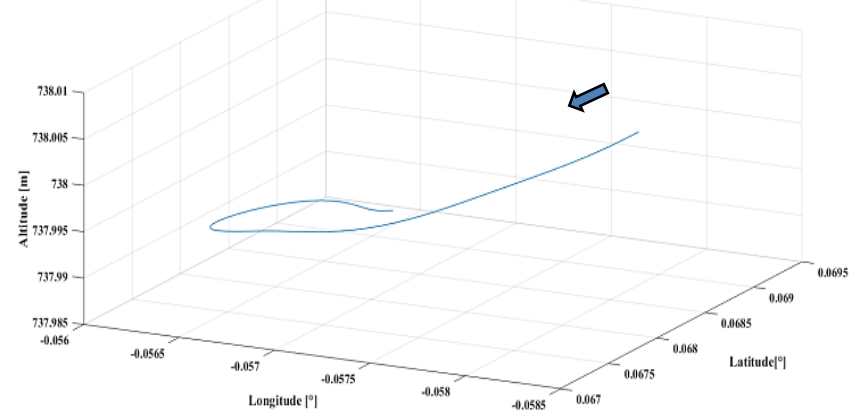

Fig. 17. TD leg segment optimised with PMO.

The trajectory obtained with the CGO technique is shown in Fig. 18. In this case, the trajectory optimisation loop took 0.42 seconds to complete in a standard PC equipped with an Intel i7 quad-core processor and 8 GB RAM.

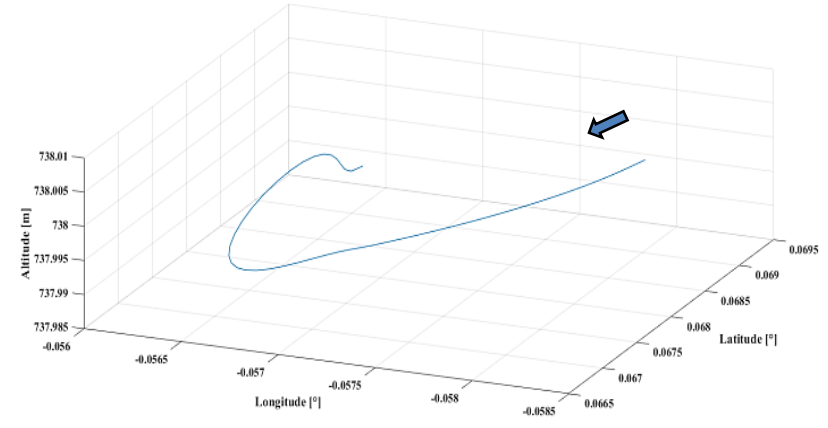

Fig. 18. TD leg segment optimised with CGO.

Further AEROSONDE simulations showed that, based on flight path length and aircraft dynamics (initial conditions), the time required for flight path optimisation varied between 5 and 220 seconds for the PMO and between 0.3 and 0.9 seconds for the CGO. Based on these results, it is evident that the PMO algorithms cannot be directly employed in real-time ABIA applications. This is because, even with the relatively benign flight dynamics of a small UAV like the AEROSONDE, the time required to perform trajectory optimisation is too long for real-time path following tasks in Automatic Flight Control System (AFCS). As already mentioned, the PMO technique is capable of generating a mathematical optimum and is better suited for ABIA mission-planning/ATM applications (i.e., ground-based and avionics mission planning tools). It is therefore concluded that the adoption of PMO/CGO techniques in the ABIA FPG module would allow an efficient exploitation of the IFG module predictive features both in mission planning and real-time trajectory optimisation problems, potentially meeting GNSS integrity requirements for ATM online operations and AFCS/ABIA integration for GLS down to CAT II/III.

\section{CONCLUSIONS AND FUTURE WORK}

In this paper we have studied the ABIA Integrity Flag Generation (IFG) and Flight Path Optimisation (FPO) modules, focussing on the potential synergies attainable by integrating ABIA functionalities into the existing GBAS and SBAS systems. The IFG module provides CIFs at least 2 second before unacceptable GNSS data degradations or signal losses (WIFs) take place. The purpose of the IFG module is to exploit these predictive features allowing the aircraft to correct its flight trajectory/attitude and to avoid the occurrence of GNSS integrity events. Analysing the various causes of GNSS data degradation or loss, geometric/mission optimisation criteria were introduced in terms of satellite elevation angle and heading rates. Adopting these criteria and adequate forms of the aircraft dynamics model (3DOF with variable mass and constraints/initial conditions set by a manoeuvre recognition algorithms), two different approaches were introduced to solve the guidance problem (i.e., generation of optimal flight trajectories) both in mission-planning and real-time ABIA applications. The approach proposed for mission planning employs a classical Pseudospectral Multi-Objective (PMO) technique and the real-time optimisation is accomplished by a Constrained Geometric Optimisation (CGO) method. Simulation case studies were accomplished on the AEROSONDE UAV to verify the suitability of the proposed techniques in a realistic operational scenario (including complex flight manoeuvres and GLS precision approach legs). The PMO technique converged to a mathematical optimum within 5 - 220 seconds depending on the length and complexity of the flight path to be optimised, while the CGO technique was able to generate trajectories free from GNSS data degradations/losses within $0.3-0.9$ seconds. SBAS and GBAS simulators were employed (based on the applicable WAAS and LAAS standards) and simulation case studies were performed to investigate the synergies attainable from the online integration of ABIA with SBAS and GBAS. Based on the simulation case studies 
performed, it is concluded that the ABIA system works synergically with SBAS and GBAS, enhancing integrity levels in all flight phases, from initial climb to final approach. The ABIA algorithms are capable of generating suitable predictive and reactive flags (CIFs and WIFs) in the same flight phases where SBAS and GBAS are designed to operate. Therefore, the integration of ABIA with SBAS/GBAS is a clear opportunity for future research towards the development of a Space-GroundAvionics Augmentation Network (SGAAN) suitable for manned and unmanned aircraft applications and for a variety of mission-critical and safety-critical aviation applications, including UAS Sense-and-Avoid (SAA), precision approach and automatic landing. Further research is focusing on the following areas:

- Study the potential applications of ABIA to cooperative and non-cooperative UAS SAA [23].

- Extend the ABAS/ABIA concept to other Communication, Navigation and Surveillance/Air Traffic Management (CNS/ATM) systems for Performance Based Operations (PBO) and 4D trajectory management [24-26].

- Investigate the potential of ABAS/ABIA techniques to enhance GNSS integrity in aircraft surface operations [27].

- Investigate the potential of ABAS/ABIA concepts to support aviation forensic applications (i.e., accident and incident investigation).

- Assess the potential synergies between ABIA and RAIM techniques, including enhanced RAIM (eRAIM) and predictive RAIM (pRAIM) in a multiconstellation GNSS environment.

\section{REFERENCES}

[1] R. Sabatini, T. Moore, and C. Hill, "A New Avionics Based GNSS Integrity Augmentation System: Part 1 - Fundamentals," Journal of Navigation, vol. 66, no. 3, pp. 363-383, May 2013. DOI: $10.1017 / \mathrm{S} 0373463313000027$

[2] R. Sabatini, T. Moore, and C. Hill, "A New Avionics Based GNSS Integrity Augmentation System: Part 2 - Integrity Flags," Journal of Navigation, vol. 66, no. 4, pp. 511-522, June 2013. DOI: $10.1017 / \mathrm{S} 0373463313000143$

[3] R. Sabatini, T. Moore, C. Hill and S. Ramasamy, "Investigation of GNSS Integrity Augmentation Synergies with Unmanned Aircraft Sense-and-Avoid Systems", SAE Technical Paper 2015-01-2456, SAE 2015 AeroTech Congress \& Exhibition, Seattle, Washington, USA, 2015. DOI: 10.4271/2015-012456

[4] R. Sabatini and G. Palmerini, "Differential Global Positioning System (DGPS) for Flight Testing," NATO Research and Technology Organization
(RTO) - Systems Concepts and Integration Panel (SCI), AGARDograph Series RTO-AG-160, Vol. 21, Oct 2008. URL: www.dtic.mil/get-trdoc/pdf?AD= ADA 493532

[5] R. Sabatini, T. Moore and C. Hill, "Avionics-Based GNSS Integrity Augmentation for Unmanned Aerial Systems Sense-and-Avoid," Proceedings of $27^{\text {th }}$ International Technical Meeting of the Satellite Division of the Institute of Navigation: ION GNSS+ 2014, Tampa, Florida, USA, 2014.

[6] W. F. Phillips, "Mechanics of Flight" John Wiley \& Sons, 2004.

[7] A. Valenzuela, and D. Rivas, "Conflict Resolution in Converging Air Traffic Using Trajectory Patterns", Journal of Guidance, Control, and Dynamics, Vol. 34, no. 4, 2011, pp. 1172-1189.

[8] Rao, A.V. (2010-b), "Trajectory Optimization”, In Encyclopedia of Aerospace Engineering, John Wiley $\&$ Sons Ltd.

[9] M. P. Hansen, "Tabu Search Multiobjective Optimisation: MOTS", in proceedings of $13^{\text {th }}$ International Conference on Multiple Criteria Decision Making (MCDM '97), Cape Town, South Africa, 1996

[10] J. A. Lennon and E. M. Atkins, "Preference-Based Trajectory Generation", in proceedings of AIAA Infotech@Aerospace 2007 (I@A 2007), Rohnert Park, CA, USA, 2007. DOI: 10.2514/6.2007-2973

[11] A. V. Rao, "Survey of Numerical Methods for Optimal Control", Advances in the Astronautical Sciences, vol. 135, pp. 497-528, 2010

[12] S. Ramasamy, R. Sabatini and A. Gardi, "LIDAR Obstacle Warning and Avoidance System for Unmanned Aerial Vehicle Sense-and-Avoid", Aerospace Science and Technology, vol. 55, 2016.

[13] Gardi, A., Sabatini, R., Ramasamy, S. (2016), "Multi-Objecive Four Dimesional Trajectory Optimisation in the ATM Context", Progress in the Aerospace Sciences, April 2016. DOI: 10.1016/j.paerosci.2015.11.006

[14] Delahaye, D., Puechmorel, S., Tsiotras, P., and Feron, E., "Mathematical Models for Aircraft Trajectory Design: A Survey", Lecture Notes in Electrical Engineering, Vol. 290, Air Traffic management and Systems, pp. 205-247, 2014

[15] RTCA, DO-229D - Minimum Operational Performance Standards for Global Positioning System/Wide Area Augmentation System Airborne Equipment, Washington DC, USA, 2006.

[16] RTCA, DO-245A - Minimum Aviation System Performance Standards for Local Area Augmentation System (LAAS), Washington DC, USA, 2004. 
[17] RTCA DO-253C - Minimum Operational Performance Standards for GPS/GBAS Airborne Equipment, Radio Technical Commission for Aeronautics, Special Committee No. 159. Washington DC (USA), December 2008.

[18] RTCA DO-246D - GNSS-Based Precision Approach Local Area Augmentation System (LAAS) Signalin-Space Interface Control Document (ICD), Washington DC, USA, 2008.

[19] FAA, Global Positioning System Wide Area Augmentation System (WAAS) Performance Standard, Washington DC (USA), 2008.

[20] J. Maslanik, Seminar on AEROSONDE UAV, Colorado Center for Astrodynamics Research (CCAR), University of Colorado at Boulder, 2002. Available online at: http://www2.hawaii.edu/ jmaurer/uav/\#specifications

[21] Unmanned Dynamics (2003). "Aerosim Blockset User's Guide.” Version 1.01. Unmanned Dynamics LLC, Hood River, OR (USA). Document available online at: https://people.rit.edu/pnveme/EMEM682n/ Matlab_682/aerosim_ug.pdf (accessed 3rd June 2012).

[22] M. T. Burston, R. Sabatini, R. Clothier, A. Gardi and S. Ramasamy, "Reverse Engineering of a Fixed Wing Unmanned Aircraft 6-DoF Model for Navigation and Guidance Applications." Applied Mechanics and Materials, Vol. 629, pp. 164-169, October 2014. DOI: 10.4028/www.scientific.net /AMM.629.164
[23] S. Ramasamy, R. Sabatini and A. Gardi, "Towards a Unified Approach to Cooperative and NonCooperative RPAS Detect-and- Avoid”, Fourth Australasian Unmanned Systems Conference 2014 (ACUS 2014), Melbourne, Australia, 2014. DOI: 10.13140/2.1.4841.3764A.

[24] Gardi, R. Sabatini, T. Kistan, Y. Lim, and S. Ramasamy, "4-Dimensional Trajectory Functionalities for Air Traffic Management Systems", in proceedings of Integrated Communication, Navigation and Surveillance Conference (ICNS 2015), Herndon, VA, USA, 2015. DOI: $10.1109 /$ ICNSURV.2015.7121246

[25] S. Ramasamy, R. Sabatini and A. Gardi, "Novel Flight Management Systems for Improved Safety and Sustainability in the CNS+A Context", Proceedings of Integrated Communication, Navigation and Surveillance Conference (ICNS 2015), Herndon, VA, USA, 2015. DOI: 10.1109/ICNSURV.2015.7121225

[26] S. Ramasamy and R. Sabatini, "A Unified Approach to Cooperative and Non-Cooperative Sense-and-Avoid", Proceedings of International Conference on Unmanned Aircraft Systems (ICUAS 2015), Denver, CO, USA, 2015. DOI: 10.1109/ICUAS.2015.7152360

[27] S. S. Bijjahalli, S. Ramasamy and R. Sabatini, "A GNSS Integrity Augmentation System for Sustainable Autonomous Airside Operations." 2nd International Symposium on Sustainable Aviation (ISSA 2016), Istanbul, Turkey, 2016. 